\title{
Eine europäische Sozialverfassung?
}

\author{
Von Ulrich Mückenberger, Bremen*
}

Die ,, sozialstaatlichen (vorsichtiger: sozialverfassungsrechtlichen) Elemente “ des europäischen Primärrechts, die Peter Häberle 1994 noch tentativ andeutete, finden sich 20 Jahre später in konsolidierter Form vor. Der Beitrag beleuchtet am zunehmenden Gewicht der europäischen Verfassungsprinzipien Menschenwürde, Demokratie und Solidarität, dass man der Europäischen Union (EU) heute den rechtlichen Charakter der Sozialstaatlichkeit - wenn auch durch die Governance eines Mehrebenen-Systems geformt - kaum mehr wird abstreiten können. Dieser juristische Befund wird zahlreichen sozialpolitischen Kontroversen, die um das Verhältnis von Marktfreiheiten und sozialen Standards auf EU- und Mitgliedstaats-Ebene geführt werden, neue normative Konturen und primärrechtliche Bewertungsmaßstäbe geben.

\section{Ein Staatenverbund mit normativer Kontur}

Die EU als Staatenverbund ${ }^{1}$ unterscheidet sich von anderen Staatenbünden und Weltregionen durch Zweierlei: Sie verfolgt erstens - neben anderen - eigene soziale Ziele und Aufgaben, denen sie auch supranationale Geltungs- und Durchsetzungsbedingungen verschafft. Sie gilt nicht nur (wie NAFTA oder ASEAN) fremden sozialen Zielen, die die jeweilige nationale Souveränität unangetastet lassen. Diese eigenen sozialen Ziele schlagen sich im EU-Primär- und Sekundärrecht nieder (dazu mehr unter Abschnitt 6), aber auch in der Aktivität gegenüber Europarat ${ }^{2}$ und dritten Staaten (dazu Titel V EUV). Die EU setzt sich zweitens aus Mitgliedstaaten zusammen, die - neben anderen - soziale Ziele und Aufgaben verfolgen und die bei aller Diversität eine nennenswerte Schnittmenge gemeinsamer Verfassungstraditionen (Art. 6 Abs. 3 EUV) sozialer Art aufweisen. Diese gemeinsame Schnittmenge lässt sich an drei Merkmalen nachweisen ${ }^{3}$ :

- Ein relativ (etwa im Vergleich zu den USA) starker sozial schützender und ermöglichender Staat;

- eine relativ (etwa im Vergleich zu Asien) starke Rolle von Individuen und Individualrechten;

\footnotetext{
* Prof. Dr. Ulrich Mückenberger ist Rechts- und Politikwissenschaftler und emeritierter Professor für Arbeits- und Sozialrecht am Fachbereich Sozialökonomie der Universität Hamburg, derzeit Forschungsprofessor an der Universität Bremen Zentrum für Europäische Rechtspolitik (ZERP) und DFG-Sfb 597 „Staatlichkeit im Wandel“.

1 BVerfG, Rs. 89 (Maastricht-Urteil), Slg. 1993, 155.

2 Hierzu bedeutsam Art. 6 Abs. 2 EUV, der die Beitrittspflicht der EU zur Europäische Konvention zum Schutz der Menschenrechte und Grundfreiheiten begründet.

3 Mückenberger (Hrsg.), Manifesto social Europe, European Trade Union (ETUI), 2001.
} 
- und eine relativ (etwa im Vergleich zu beiden genannten Weltregionen) starke Anerkennung des Konflikts (individuell und kollektiv) als Mittel zur Erzielung sozialen Fortschritts, sogar sozialen Zusammenhalts.

Gewiss sind die Mitgliedstaaten der EU in punkto Sozialstaatlichkeit unterscheidbar in Gruppen, wie sie Gösta Esping-Andersen ${ }^{4}$ und im Gefolge seines Ansatzes andere $^{5}$ formuliert haben: die bismarcksche deutsche und österreichische, die angelsächsische, die nordisch-sozialdemokratische und die südlich-rudimentäre Ausrichtung des Sozialstaats (etwa in Griechenland, Portugal, Italien und Spanien). Über ihrer Diversität sollte aber die im Weltmaßstab nicht zu leugnende Konvergenz dieser Gruppen nicht unterschätzt werden.

Deshalb ist auch die Rechtsprechung des EuGH, insbesondere sofern sie zu sozialen Grundrechten Bezug hatte - wie etwa die „Viking“-/,LLaval“-Rechtsprechungssequenz, die das Koalitions- und Streikrecht den binnenmarktlichen Grundfreiheiten des EU-Rechts unterordnete - bereits Ansatzpunkt einer Reflexion europäischer Sozialstaatlichkeit gewesen. ${ }^{6}$

Angesichts der genannten Ausgangspunkte kann und soll der EU-Staatenverbund auf soziale Verfasstheit abgeprüft werden. Dies kann aber nicht in direkter Analogie zur Sozialstaatlichkeit von Einzelstaaten geschehen. ${ }^{7}$ Von Anbeginn an muss bei dieser Analyse der Charakter der EU als der eines multi-tiered system of governance, als der eines Mehrebenen-Systems, berücksichtigt werden. ${ }^{8}$ Deshalb stelle ich schon im Ausgangspunkt nicht die Frage nach dem ,europäischen welfarestate“, sondern nach europäischer „,sozialer Verfasstheit“. „Welfare-state“ bezeichnet meist nur den sozial umverteilenden Staat, „Sozialstaat" demgegenüber auch den sozial gestaltenden Staat. Da zentrale Instrumente und Ressourcen der sozialen Umverteilung (etwa i. R. der staatlichen und privaten sozialen Sicherung) nicht europäisiert sind, wäre die Frage nach dem europäischen „welfare-state“ schon im

4 G. Esping-Andersen, Three Worlds of Welfare Capitalism, 1990.

5 Vgl. Lessenich/Ostner (Hrsg.), Welten des Wohlfahrtskapitalismus. Der Sozialstaat in vergleichender Perspektive, 1998; B. Pfau-Effinger, Culture and Welfare State Policies: Reflections on a Complex Interrelation, in: Journal of Social Policy, 2005, S. 1-18.

6 Vgl. etwa F. Mayer, Der EuGH und das soziale Europa, in: Friedrich-Ebert-Stiftung (Hrsg.), Der EuGH und das soziale Europa. Für eine Aufwertung sozialer Grundrechte im EU-Rechtssystem, 2009, S. 7-25 und die Kurzbeiträge in der gleichnamigen Schrift der Friedrich-Ebert-Stiftung, Der EuGH und das soziale Europa. Für eine Aufwertung sozialer Grundrechte im EU-Rechtssystem, 2009.

7 Zum Begriff der „Verfassung“ bzw. der „Konstitutionalisierung“ im Zusammenhang mit der EU beziehe ich mich v.a. auf die Schriften von Müller-Graff/Riedel (Hrsg.), Gemeinsames Verfassungsrecht in der Europäischen Union, darin: Müller-Graff/Riedel, Gemeinsames Verfassungsrecht in der Europäischen Union - Einführung, 1998, S. 9-14 und P.-C. Müller-Graff, Die wettbewerblich verfasste Marktwirtschaft als gemeineuropäischer Verfassungsprozess, 1998, S. 53-75; T. Giegerich, Europäische Verfassung und deutsche Verfassung im transnationalen Konstitutionalisierungsprozess, 2003; Bogdandy/Bast (Hrsg.), Europäisches Verfassungsrecht. Theoretische und dogmatische Grundzüge, 2009, 2. Aufl.; Tsatos (Hrsg.), Die Unionsgrundordnung - Handbuch zur Europäischen Verfassung, 2010; C. Franzius, Europäisches Verfassungsdenken, 2010; P. Häberle, Europäische Verfassungslehre, 2011, 7. Aufl.

8 Dieser Erkenntnis folgt T. Giegerich (Fn. 7), S. 5 ff., 8 ff., 12 ff. sorgfältig, indem er die „Vorgabekomponente“ von der „Projektionskomponente“ und der „Reaktionskomponente" transnationaler Konstitutionalisierung unterscheidet: Auf „Vorgaben“ der Nationalstaaten (Mitgliedstaaten) an das transnationale Recht hin folgt die Projektion transnationalen Rechts als dauerhafte institutionalisierte Kooperation, was dann wiederum die Rückwirkung (Reaktion) der transnationalen auf die nationalen Konstitutionalisierungsprozesse hervorruft. 
Ausgangspunkt falsch gestellt. ${ }^{9}$ Ich stelle auch nicht die Frage nach dem europäischen Sozial"staat", sondern nach europäischer Sozial"verfassung" oder europäischer "Sozialstaatlichkeit", und möchte damit deutlich machen, dass die Suche nicht einem bestimmten Staatstyp gilt. Sie gilt vielmehr einem - durchaus changierenden - Merkmal des gegebenen Mehrebenen-Systems der EU: Dieses System ist normativ durch eigene soziale Ziele und Aufgaben sowie durch gemeinsame soziale Ziele und Aufgaben der Mitgliedstaaten auf eine „,soziale Verfassung“ ausgerichtet. ${ }^{10}$ In ähnlichem Zugang und mit ähnlicher Terminologie hob bereits 1994 Peter Häberle die „sozialstaatlichen“ Elemente der - damals durch den Maastricht-Vertrag modernisierten - EG hervor. Er bezeichnete sie damals bezeichnenderweise als ,,sozialstaatliche (vorsichtiger: sozialverfassungsrechtliche) Elemente“. ${ }^{11}$

Die Analyse der so präzisierten Frage nach der europäischen sozialen Verfasstheit kann in fünf analytische Fragen untergegliedert werden:

- Was ist der Gegenstand der europäischen Sozialverfassung, insbesondere wenn wir sie mit dem Focus der Regulierung gesellschaftlicher Arbeit (v. a. abhängiger Erwerbsarbeit) betrachten?

- Welches sind die Methoden, derer sich die Sozialverfassung bedient?

- Auf welche Rechtsquellen beziehen wir uns dabei?

- Welche Beziehung weist das viel zitierte „Europäische Sozialmodell“ zu der hier verfolgten europäischen Sozialstaatlichkeit auf?

- Welche normative Gestalt weist die europäische Sozialverfassung auf?

\section{Gegenstände der arbeitsbezogenen europäischen Sozialverfassung}

Zunächst wird der rechtliche Gegenstandbereich umrissen, der von europäischer Sozialverfasstheit umrissen ist. Hierzu nehme ich die Einschränkung auf ,,arbeitsbezogene soziale Verfasstheit" vor. Der normative Nachweis europäischer Sozialverfasstheit wird zwar auf weitere Bereiche als „Arbeit“" Auswirkungen haben. Jedoch bedürften diese gegenständlicher Spezifikation, wie ich sie hier nur exemplarisch am Gegenstandsbereich Arbeit vornehmen kann. Wenn wir uns auf die Regelung abhängiger Arbeit konzentrieren, gehören gegenstandslogisch zur Analyse europäischer Sozialstaatlichkeit drei Bereiche: die Regelung der Arbeit betreffenden Marktbeziehungen (unten 1.); die Regelung der auf Arbeit bezogenen, aber dem

9 So zu Recht S. Huster, Eine soziale Dimension Europas, in: Tsatsos (Hrsg.) (Fn. 7), S. 369. Daraus folgt jedoch nicht zwangsläufig, dass das europäische Rahmenwerk auf ein „,europäisches Sozialmodell“ zu reduzieren sei (wie von S. Huster auf S. 378/79 vorgeschlagen). Darauf werde ich in Abschnitt 6 zurückkommen.

10 Aus den genannten Gründen werden im nachfolgenden Text die Begriffe „europäische Sozialverfassung“, „,europäische soziale Verfasstheit“, „europäische sozialstaatliche Verfasstheit“ und „europäische Sozialstaatlichkeit" nicht trennscharf, sondern i.a. im selben Sinn verwendet.

11 P. Häberle, Europäische Rechtskultur. Versuch einer Annäherung in zwölf Schritten, 1994, 1. Aufl., S. 86 ff. Häberle fügt denn auch sogleich hinzu: „Kernbereiche der Sozialstaatlichkeit bleiben in der Rechtsgemeinschaft der EG - noch - ausgeblendet.“, S. 91. Siehe auch das von Bast und Rödl herausgegebene EuR-Beiheft 1/2013 "Wohlfahrtsstaatlichkeit und soziale Demokratie in der Europäischen Union", ferner C. Nowak, "Wettbewerb und soziale Marktwirtschaft in den Regeln des Lissaboner Vertrages", EuR, Beiheft 2/2011, S. 21 ff., auf die im Folgenden stellenweise eingegangen wird. 
Markt entzogenen staatlichen Sicherungen (unten 2.); und die Regelung der gesellschaftlichen Effekte, die mit der Marktförmigkeit des Austauschs von Arbeit verbunden sind (unten 3.).

\section{Arbeitsrecht}

Arbeitsrecht wird verstanden als Regelung der Erwerbsarbeit in Arbeitsverhältnissen und ähnlicher abhängiger Form. ${ }^{12}$ Regulierung hat hier zivile (individuelle und kollektive) und soziale (individuelle und kollektive) Gegenstände. ${ }^{13}$ Geregelt wird in der Sozialverfassung insoweit ein Doppeltes: Dass hinsichtlich Arbeit überhaupt verlässliche Marktbeziehungen möglich sind und dass diese Marktbeziehungen Kriterien sozialer Gerechtigkeit entsprechen. Für diese Doppelfunktion haben sich in der Literatur die Begriffe der Arbeitsmarktherstellung und -sicherung einerseits, der Marktkorrektur andererseits herausgebildet. ${ }^{14}$ Rechtlich geschieht Arbeitsmarktherstellung individuell durch Gewährleistung von Arbeitsvertragsfreiheit (Befreiung von Zwang wie Leibeigenschaft - inkl. Sicherung gegen Marktversagen durch Diskriminierung, unmittelbaren Zwang, externe Effekte etc.) und kollektiv durch Gewährleistung von Tarifvertragsfreiheit. Beides zusammen bewirkt das, was Esping-Andersen aus sozialpolitischer Perspektive als ,commodification“ (also die Tauschförmigkeit) von Arbeit bezeichnet hat. ${ }^{15}$ Arbeitsmarktkorrektur geschieht rechtlich gleichfalls sowohl individuell als auch kollektiv. Das Arbeitsrecht setzt individuell meist zumindest einseitig zwingendes Schutzrecht. Es fördert kollektiv soziale Dialoge und Aushandlungssysteme zwischen den Sozialpartnern und kontrolliert und gewährleistet deren Resultate.

\section{Sozialrecht}

Sozialrecht ist zu verstehen als die marktergänzende öffentlich-rechtliche Regelung solcher Elemente von Arbeitsbeziehungen, die sich privatrechtlicher, marktförmiger Regulierung entziehen. ${ }^{16}$ Regulierung hat hier eine zweifache Form. ${ }^{17}$ Sie be-

12 Dazu mit europäischer Perspektive M. Schlachter/A. Seifert, Arbeitsrecht, in: Schulze/Zuleeg/Kadelbach (Hrsg.), Europarecht. Handbuch für die deutsche Rechtspraxis, 2010, 2. Aufl., S. 2066-2126; ferner F. Rödl, Arbeitsverfassung, 2009, in: Bogdandy/Bast (Hrsg.) (Fn. 7), S. 855-904.

$13 \mathrm{Zu}$ Grunde gelegt wird bei der Begrifflichkeit das Konzept von T. H. Marshall, Citizenship and Social Class and Other Essays, 1950. Marshall unterscheidet zwischen civil, political und social rights of citizenship. Interessant ist dabei, was Marshall als „industrial citizenship“ bezeichnet: Erst aufgrund von Demokratisierung (=political rights) erreichen die an sich individuellen ,civil rights“ die Form einer kollektiven, darum ,,industrial“ genannten citizenship (=Koalitionsfreiheit, Tarifvertragsfreiheit).

14 Bei marktbezogenen Regulierungsfunktionen gehe ich von der Trias market-making (rechtliche Herstellung des marktförmigen Austausches), market-braking (Sicherung des Marktprozesses gegen Marktversagen: externe Effekte, Kartelle, marktwidrige Stigmatisierung und Diskriminierung) und market-correcting (rechtliche Korrektur marktförmiger Aushandlungsergebnisse nach Kriterien der sozialen Gerechtigkeit und Sozialverträglichkeit) aus.

15 G. Esping-Andersen (Fn. 4).

16 Dazu mit europäischer Perspektive E. Eichenhofer, Sozialrecht der Europäischen Union, 2010, 4. Aufl.; H. D. Steinmeyer, Sozialrecht, in: Schulze/Zuleeg/Kadelbach (Hrsg.) (Fn. 12), S. 2127-2214.

17 E. Eichenhofer (Fn. 16). 
steht einerseits in der Vorsorge gegenüber solchen arbeitsbezogenen Risiken, die der Marktteilnahme der betreffenden Person vorübergehend oder dauerhaft im Wege stehen (z.B. durch Maßnahmen der Sozialversicherung). Dies hat Esping-Andersen in seiner sozialpolitischen Typologie als ,decommodification“" (also die partielle Aufhebung von Tauschförmigkeit) bezeichnet. ${ }^{18}$ Sozialrechtliche Regulierung besteht andererseits in staatlicher Hilfe/Fürsorge. Diese soll auf nicht-marktförmige Weise sicherstellen, dass die betroffene Person wenn schon nicht am Arbeits-, so doch am Konsumgütermarkt teilnehmen kann (z.B. mittels Sozialhilfe). Arbeitsrecht ist im Prinzip Privatrecht, hat aber öffentlich-rechtliche Anteile (Arbeitsschutz, Betriebsverfassung, normativer Teil des Tarifvertrages). Sozialrecht ist im Prinzip öffentliches Recht, hat aber privatrechtliche Bezugspunkte (was sich z.B. in Sperrzeiten- und Beschäftigungsfähigkeitsbestimmungen im Arbeitsförderungsoder im Lohnabstandsgebot im Sozialhilferecht niederschlägt).

\section{Regulierung gesellschaftlicher Effekte}

Der dritte Bestandteil von arbeitsbezogener Sozialverfassung ist ein weniger geläufiges und konsolidiertes, aber wichtiger werdendes Rechtsgebiet. Reguliert werden hier gesellschaftliche Voraussetzungen und gesellschaftliche (bes. unerwünschte) Folgen von Arbeitsmarktprozessen. Diese Regelungsbereiche fallen im deutschen Sprachgebrauch gleichfalls unter den Begriff des „Sozialen“. Klarer ist insoweit die englische Sprache, die diese Bereiche als "societal" (gesellschaftlich) bezeichnet und damit von ,social“" (sozial) abhebt. Beide genannten Regelungsbereiche der Sozialverfassung liegen weithin außerhalb der Regulierungskapazität und -befugnis der privaten Arbeitsvertrags- und Kollektivvertragsparteien. Gesellschaftliche Voraussetzungen von Arbeitsmarktprozessen sind z.B. Berufliche Bildung, räumliche Infrastruktur, soziale Infrastruktur zur Vereinbarkeit von Erwerbsarbeit und Lebenssituation, öffentliche Wirtschaft, Auftragsvergabewesen. Gesellschaftliche Folgen der Arbeitsmarktprozesse können erwünscht sein (z.B. Erhöhung des Bruttosozialprodukts, Wohlstandsmehrung etc.). Sie können aber auch unerwünscht sein (z.B. negative externe Effekte, produktionsbedingte Umweltschäden, soziale Spaltung und Diskriminierung, geographische Disparitäten, kulturelle Prägungen - z.B. Egoismus und Konkurrenzdenken, Produktivismus und darin implizierte Kinderfeindlichkeit). Hier öffnet sich ein weites Gestaltungsfeld.

Die Regelungsgebiete, in denen gesellschaftliche Voraussetzungen und Folgen geregelt werden, sind denn auch vielfältig (z.B. Umweltrecht, Berufsbildungsrecht, Vergaberecht, Kinder- und Jugendhilferecht, Recht des Länderfinanzausgleichs und des ESF etc.). Sie werden von Arbeits- und Sozialrecht i. A. getrennt gedacht und verfasst. In gesellschaftlichen Aufschwungs- und Wohlstandsphasen (z.B. den, ,goldenen 1950er/1960er Jahren“") treten sie deshalb meist hinter den zwei zuvor genannten zurück. Deshalb ist dieses Rechtsgebiet wenig konsolidiert. In Krisen- und 
Umbruchphasen wie der heutigen gewinnen sie dagegen wieder an Bedeutung. Sie werden so auch Gegenstand von sozialer Verfassung.

\section{Methoden sozialer Verfassung im Mehrebenen-System}

Im nationalen Rahmen hat die Regulierung der drei o. g. Gegenstandsbereiche einen langen rechtstraditionalen Vorlauf. Kernmethode der Normbildung ist das vom Parlament auf Grundlage der Verfassung verabschiedete „Gesetz“. Dieses kann direkte arbeitsbezogene Normen setzen. Es kann indirekte, die Selbstregulierungsbefugnis der Betroffenen stärkende Regelungen treffen. Es kann die Regulierungsbefugnis bewusst für die Betroffenen offenhalten (z.B. durch Einräumung von Autonomien wie die Koalitionsfreiheit). Ob vom Regulierungsmittel Gesetz Gebrauch gemacht wird oder nicht, kann allgemeinpolitisch begründet sein (z.B. gestützt auf die Regulierungs- oder die Deregulierungsschule). Es kann auch bereits arbeitsbezogene Gründe haben: So wird den Tarifvertragsparteien im deutschen und italienischen Recht eine Normsetzungsprärogative eingeräumt, wie sie im französischen oder spanischen Arbeitsrecht nicht zu finden ist.

Im nationalen Rahmen werfen föderale Strukturen kein wirkliches Kompetenzproblem für staatliche Regulierung auf. Auch Bundesstaaten verfügen über ungeteilte Souveränität und damit über eine eindeutige „Kompetenz-Kompetenz“ (für Deutschland Art. 31 GG). Deshalb ist die Abgrenzung von Bundes- und Landeskompetenzen eher technischer als politischer Natur.

Ganz anders verhält es sich mit der arbeitsbezogenen Verfassung der Europäischen Union. Das Primärrecht der EU hat - durch das Subsidiaritätsprinzip (Art. 5 Abs. 3 EUV) eingehegt - Befugnisse zur Normbildung auf die EU übertragen und damit zu geteilter Souveränität geführt. Der relativ kurze rechtstraditionale Vorlauf fand unter Bedingungen großer nationaler Diversität der Arbeitskulturen der Mitgliedstaaten statt. Im Vordergrund und historischen Ausgangspunkt der europäischen Einigung stand, was Fritz Scharpf als „negative Integration“19 bezeichnete: Die marktherstellende Integration, die - i.A. Alltagsgeschäft von Kommission und Rat - meist rasch und ohne öffentliches Aufsehen verlief. Negative Integration hatte fast immer Vorsprung und Übergewicht gegenüber ,positiver Integration“. Damit bezeichnete Scharpf die marktkorrigierende Integration, die - i.A. der intergouvernementalen Ebene vorbehalten - immer zögerlich und unter öffentlichem Aufsehen geschah. Dass sie diesen Spezifika positiver Integration zugehört, erklärt, warum europäische soziale Regulierung eher einen Flickenteppich als ein konsolidiertes Ganzes darstellt. ${ }^{20}$

19 Terminologie und Konzept lehnen sich an F. Scharpf, Negative and Positive Integration in the Political Economy of European Welfare States, in: Marks/Scharpf/Schnitter/Streeck (Hrsg.), Governance in the European Union, 1996, S. 15-39.

20 Dieser europäische Rechtsrahmen hat durch die Folgen der Finanzmarkt- und Euro-Krise noch an Konsistenz (auch an Legitimität?) verloren. Über Koalitionsfreiheit zu sprechen, ist heute (angesichts der Politik der Troika) in Portugal oder Griechenland etwas definitiv anderes als in Schweden oder Deutschland. 
Die Mehrebenen-Governance erschwert die Ermittlung der Methoden, mit denen europäische Sozialverfassung hergestellt wird. Wie alle europäische Regulierung unterliegt auch die soziale den Prinzipien der enumerativen Einzelermächtigung (Art. 5 Abs. 2 EUV) ${ }^{21}$ und der Subsidiarität (Art. 5 Abs. 3 EUV). ${ }^{22}$ Die EU darf nur regeln, wenn sie dazu explizit und bestimmt durch Primärrecht befugt ist. Wo sie und die Mitgliedstaaten gleichermaßen normsetzungsbefugt sind, ist nur die geeignetere Ebene zur Normsetzung befugt. Wo beide Hürden überwunden und die EU zur Normsetzung befugt ist, setzt sie „supranationales“ Recht - d.h. Recht, das unmittelbar in den Mitgliedsstaaten gilt, das Vorrang selbst vor ranghöchstem Recht der Mitgliedstaaten hat und dessen Durchführung durch alle Beteiligte dem ,effet utile“ (EuGH) folgen muss. ${ }^{23}$

Vor diesem rechtstechnischen Hintergrund sind drei Methoden der Herstellung europäischer sozialer Verfasstheit im Mehrebenen-System zu unterschieden. Bei der „Harmonisierung“ von Arbeits- und Sozialrecht gleichen europäische Instanzen die nationalen Rechte in Richtung auf einen europäischen Maßstab an (unten 1). Dagegen wird bei der „Koordinierung“ arbeitsbezogenen Rechts diese Angleichung zwar durch Europa angeregt, ihr Ob und Wie verbleibt aber in der Machtvollkommenheit der Mitgliedstaaten (unten 2). Soziale, regionale sowie globale „Strukturpolitik" schließlich verfolgt kein Angleichungsziel, sondern versucht, nationale und regionale Unterschiedlichkeiten durch europäische Beihilfe- und Ausgleichsmaßnahmen zu kompensieren (unten 3). ${ }^{24}$

\section{Harmonisierung}

Harmonisierung ist die ursprüngliche Methode der Erzeugung von Normen auf dem Gebiet europäischer Sozialstaatlichkeit. Von ihr war bereits in den EWG-Verträgen als „Aufwärts-Harmonisierung“ programmatisch die Rede. Soziale Angleichung sollte eine Angleichung ,nach oben“ sein - vom ursprünglichen Art. 117 EWGVertrag bis zum heute geltenden Art. 151 AEUV gilt als primärrechtliches Ziel, ,auf dem Wege des Fortschritts“ diese ihre Angleichung zu ermöglichen. Die Harmonisierung folgt dabei der sog. „Gemeinschaftsmethode“ (,,community method“). ${ }^{25}$ Aufgrund der durch bestehendes Primärrecht erteilten Ermächtigung ergreift die

21 Dazu allgemein C. Calliess in: Calliess/Ruffert (Hrsg.), EUV/AEUV-Kommentar, 2011, 4. Aufl., Art. 5 Abs. 2 EUV, Rn. 6 ff.; M. Zuleeg/S. Kadelbach in: Schulze/Zuleeg/Kadelbach (Hrsg.), Europarecht. Handbuch für die deutsche Rechtspraxis, 2010, 2. Aufl., § 8, Rn. 43; G. Lienbacher in: Schwarze (Hrsg.), EU-Kommentar, 2012, 3. Aufl., Art. 5 Abs. 2 EUV, Rn. 6; R. Streinz in: Streinz (Hrsg.), EUV/AEUV-Kommentar, 2012, 2. Aufl., Art. 5 Abs. 2 EUV, Rn. 8 ff.

22 Dazu allgemein C. Calliess in: Calliess/Ruffert (Fn. 21), Art. 5 Abs. 3 EUV, Rn. 20 ff.; M. Nettesheim in: Oppermann/Classen/Nettesheim, Europarecht, 2011, 5. Aufl., § 11, Rn. 23 ff.; M. Zuleeg/S. Kadelbach in: Schulze/ Zuleeg/Kadelbach (Fn. 22), § 2, Rn. 28; G. Lienbacher in: Schwarze (Fn. 21), Art. 5 Abs. 3 EUV Rn. 15 ff.; $R$. Streinz in: Streinz (Fn. 21), Art. 5 Abs. 3 EUV Rn. 19 ff.

23 Dazu die Angaben in Fn 22.

24 Die folgende Darstellung weicht von der Zweiteilung bei E. Eichenhofer (Fn. 16) insofern ab, als dieser die OMK der Harmonisierungsmethode zuordnet (S. 286 ff.) und die dritte Methode nicht ausweist.

25 Dazu grundlegend G. Majone, The European Community between Social Policy and Social Regulation, Journal of Common Market Studies, 1993, S. 153-175. 
Kommission die Initiative zu einer sekundärrechtlichen Regelung. Diese wird im Rat und im Europäischen Parlament beraten und nach Normsetzungsmodus und Stimmgewichtungsmodalitäten der Verträge beschlossen. Wie die so beschlossene Norm in den Mitgliedstaaten gilt, richtet sich nach der Form des gewählten Rechtsinstruments (Art. 288 Abs. 1 AEUV). Im Falle der Verordnung gilt die beschlossene Norm unmittelbar in den Mitgliedstaaten (Art. 288 Abs. 2 AEUV) - so i.A. sozialrechtliche europäischen Regelungen. Im Falle der Richtlinie gilt zwar ihr Ziel unmittelbar, die Wahl der Form und Mittel bleibt aber den Mitgliedsstaaten überlassen (Art. 288 Abs. 3 AEUV), wobei bei der EuGH über den „effet utile“ der gewählten Umsetzung wacht - so i.A. der arbeitsrechtliche Typus europäischer Regulierung. Die Methode der Harmonisierung hat bereits substantielle Schritte zur Herstellung europäischer Sozialstaatlichkeit erbracht. Dazu gehören Maßnahmen etwa zur Herstellung und Verbreiterung der Freizügigkeit der Arbeitnehmer - einschl. derjenigen der Familienangehörigen und der Anerkennung von Berufszertifikaten, zur sozialen Sicherung von sog. Wanderarbeitnehmern, zur geschlechtlichen Nicht-Diskriminierung, zum Arbeitnehmerschutz bei Massenentlassung, Betriebsübergang und Unternehmensinsolvenz, zum Arbeits- und Gesundheitsschutz und zur kollektiven Interessenvertretung in Betrieb und Unternehmen usw. ${ }^{26}$

Ein Sonderfall sozialer Harmonisierung durch die Gemeinschaftsmethode ist heute in Art. 154/155 AEUV niedergelegt. Kommissionspräsident Jacques Delors hatte in den 80er Jahren, im Gefolge der Val Duchesse-Gespräche, den Sozialen Dialog (SD) entdeckt und entwickelt. ${ }^{27}$ Das Sozialprotokoll von Maastricht (1991/93), 1997 in den Amsterdamer Vertrag integriert, etablierte die soziale Normsetzungsbefugnis des SD. Dabei vereinbaren die Sozialpartner - anstelle von Kommission, Rat und EP - Normen, die sie dann entweder in den Mitgliedsstaaten autonom umsetzen oder die durch Ratsbeschluss (ohne das EP) in EU-Sekundärrecht inkorporiert werden. Ersterer Fall folgt nicht mehr der Gemeinschaftsmethode, wohl aber der zweite Fall. Er überlässt zwar die Ausformulierung der zu generierenden Normen den Sozialpartnern. Aber erst der inkorporierende Ratsbeschluss verleiht diesen Normen allgemeine, über den Einflussbereich der Sozialpartner hinausreichende rechtliche Geltung in den Mitgliedstaaten. Diese Regulierungsmethode beruht selbst historisch auf einem Entwurf des SD. ${ }^{28}$ Ihr folgten zu Beginn zahlreiche SDVereinbarungen (z.B. atypische Beschäftigung, Elternurlaub).

26 Dazu M. Schlachter/A. Seifert, (Fn. 12).

27 Zum Sozialen Dialog in Europa s. U. Mückenberger, Towards Procedural Regulation of Labour Law in Europe: The Case of Social dialogue, in: Magnusson/Strath (Hrsg.), A European Social citizenship? Preconditions for Future Policies from a Historical Perspective, 2004, S. 273-294; ferner U. Mückenberger/K. Nebe, Formwandel von Staatlichkeit durch transnationalen sozialen Dialog. Ein Forschungsvorhaben, Zeitschrift für ausländisches und internationales Arbeits- und Sozialrecht (ZIAS), 2013, S. 82-104; rechtsvergleichend M. Rhodes, Employment Policy, in: Wallace/Pollack/Young (Hrsg.), Policy-Making in the European Union, 2005, 5. Aufl., S. 279-304; U. Mückenberger, Workers' Representation at Plant and Enterprise Level, in: Hepple/Veneziani (Hrsg.), The Transformation of Labour Law in Europe. A Comparative Study of 15 Countries 1945-2004, 2009, S. 233-262.

28 M. Rhodes (Fn. 27). 


\section{Koordinierung}

Koordinierung als Methode erzeugt nicht europäische Normen. Vielmehr koordiniert sie Politik und Normsetzung der Mitgliedstaaten (MS) auf Feldern der Sozialstaatlichkeit. Eichenhofer hat darauf hingewiesen, dass Koordinierung keine neue Methode sei. ${ }^{29}$ Jedoch hat sie erstmals mit dem „Beschäftigungskapitel“ des Amsterdamer Vertrages - als „offene Methode der Koordination“ (OMK) ${ }^{30}$ - eine Stellung im Primärrecht erlangt, die seither auch auf andere Felder ausgeweitet worden ist.

Im Gegensatz zur Gemeinschaftsmethode haben bei der Koordinierungsmethode Kommission und Rat eine moderierende und proklamierende, nicht eine normbildende Rolle; EP und EuGH kommen darin nicht vor. Kommission und Rat formulieren einen Themenkomplex, zu dem MS (u.U. in regelmäßigen Abständen) in Form von Länderberichten Stellung nehmen. Ein von der Kommission benannter Expertenkreis wertet die Berichte nach best practice-Gesichtspunkten aus und entwirft Leitlinien für den weiteren nationalen Umgang mit dem Themenkomplex. Rat und Europäischer Rat verkünden diese Leitlinien. Dann beginnt ggf. der erneute Berichtszyklus. Die MS sind zur Berichterstattung, nicht aber zur Einhaltung der Leitlinien oder der sie betreffenden Empfehlungen verpflichtet.

Der Beitrag der OMK zur europäischen Sozialverfassung ist ambivalent. Gewiss werden dabei arbeitsbezogene Gegenstände, die vorher allein nationale Angelegenheit waren, zum Gegenstand europäischen Koordinierens. Gewiss werden mit verabschiedeten Leitlinien bestimmte EU-gemeinsame normative Vorstellungen bekannt gemacht und zum EU-gemeinsamen Ziel erhoben. Normen im rechtlichen Sinne schafft Koordinierung aber nicht, will sie auch nicht schaffen. Sie behält wirkliche Normbildung den MS vor. Deshalb ist die Methode der Koordinierung systematisch dem Inter- oder Transgouvernementalismus zuzuordnen. ${ }^{31}$ Im Unterschied zur Methode der Harmonisierung lässt sie die MS-Souveränität unangetastet; den für Schaffung und Überwachung supranationalen Rechts verantwortlichen Institutionen (Kommission, Rat, EP, EuGH) entzieht sie insoweit ihre Funktion.

Anwendungsbereiche der OMK sind bislang die Felder Beschäftigung, Berufsbildung, Alterssicherung und soziale Ausgrenzung etc. Diese Problemfelder sind damit normativ - wenn auch nicht regulatorisch - als für europäische Sozialstaatlichkeit bedeutsam eingestuft worden.

29 E. Eichenhofer (Fn. 16).

30 B. Hepple, Labour Law and the Global Trade, 2005.

31 Transgouvernementalismus besteht nach überkommenem völkerrechtlichen Muster in der Politikkoordination von Staaten ohne auch nur partielle Souveränitätspreisgabe: zum Bedeutungsgewinn dieses policy mode innerhalb der EU s. H. Wallace, An Institutional Anatomy and Five Policy Modes, in: Wallace/Pollack/Young (Hrsg.), Policy-Making in the European Union, 2010, 6. Aufl., S. 69-104. 


\section{Strukturpolitik}

Europäische Strukturpolitik ist das Feld der sog. "Strukturfonds." 32 Von diesen Fonds - dem Europäischen Ausrichtungs- und Garantiefonds für die Landwirtschaft, dem Europäischen Sozialfonds, dem Europäischen Fonds für regionale Entwicklung (Art. 175 AEUV) - ist der Europäische Sozialfonds (ESF) der älteste (Art. 162-164 AEUV). Hinzugetreten sind der europäische Regionalfonds (Art. 176 AEUV), der der Infrastruktur dienende Kohäsionsfonds (Art. 177 Abs. 2 AEUV), daneben der nach der „Reservebefugnis“ des Art. 175 Abs. 3 AEUV eingerichtete Globalisierungsfonds.

Die Arbeit der Fonds folgt den in Art. 174 AEUV aufgeführten ,Zielen“: „Die Union entwickelt und verfolgt weiterhin ihre Politik zur Stärkung ihres wirtschaftlichen, sozialen und territorialen Zusammenhalts, um eine harmonische Entwicklung der Union als Ganzes zu fördern. Die Union setzt sich insbesondere zum Ziel, die Unterschiede im Entwicklungsstand der verschiedenen Regionen und den Rückstand der am stärksten benachteiligten Gebiete zu verringern. “

Weder verfolgen die Fonds ein normatives Angleichungsziel noch bedienen sie sich regulativer Mittel. Gespeist aus dem EU-Haushalt vergeben sie i.A. (Komplementär-) Subventionen nach Kriterien sozialer Unterstützungsbedürftigkeit - sie unterstützen Objekte, die als unterstützungsbedürftig eingestuft sind. Sie kreieren dabei materielle Anreize für die einschlägigen MS, diese Objekte gleichfalls zu unterstützen. Dabei folgen sie einem Stufensystem (z.B. „Ziel 1 ...“: Mezzogiorno, Irland, Ex-DDR), das wissenschaftlich gestützt wird, Unterstützungsbedürftigkeit festlegt und bei dem die Komplementärbeteiligung der betroffenen MS abgestuft erhöht oder vermindert - wird. Die Fonds werden von der Kommission mit Unterstützung eines drittelparitätischen Ausschusses administriert. Subventionen fließen in dezentrale Entwicklungs-, Arbeitsbeschaffungs-, Berufsbildungs- und berufliche Anpassungsprozesse.

Auch Strukturpolitik schafft also einen Beitrag zu europäischer Sozialverfassung. Europäische Institutionen machen arbeitsbezogene Gegenstände (z.B. Wirtschaftsund Einkommensdisparitäten) zum Gegenstand europäischen Agierens und formulieren dabei normative Ziele. Die Tatsache der Komplementärförderung setzt auch für MS bestimmte normative soziale Vorgaben und fördert sie. Eine sozialstaatliche Regulierungsfunktion kommt der Strukturpolitik zwar nicht zu. Aber durch ihre bloße Existenz, durch die Fixierung von Themen und Förderungsrichtungen festigt sie die normative Vorstellung einer ,Gleichheitlichkeit von Lebensbedingungen in der EU“ und der Legitimität und Notwendigkeit entsprechender Marktkorrektur nach Kriterien sozialer Gerechtigkeit (market-correcting). Auch wenn diese normative Vorstellung allgemein bleibt und konkretisierender Regulation entbehrt,

$32 \mathrm{Zu}$ den Fonds und der Europäischen Investitionsbank s. etwa K. A. Schachtschneider, Verfassungsrecht der Europäischen Union, Teil 2: Wirtschaftsverfassung mit Welthandelsordnung, 2010, S. 216-227. 
bleibt sie ein allgemeiner Referenzpunkt eines Gebots sozialstaatlichen Ausgleichs, dem Gleichheit als Ziel und Solidarität als Mittel zu ihrer Herstellung dienen.

\section{Rechtsquellen europäischer Sozialverfassung}

Bei der Suche nach der Begründung einer europäischen Sozialverfassung orientieren wir uns zunächst am Primär- und Sekundärrecht der EU. Diese partizipieren am Vorrang des Gemeinschaftsrechts und sind daher gerade für eine Extrapolation von Staatszielen bedeutsam. Dazu gehören v. a. die Grundsatzbestimmungen des EUV (vornehmlich Art. 2, 3 und 6 EUV), die Eingangsbestimmungen (vornehmlich Art. 7 bis 17 AEUV) sowie Sozial- und Beschäftigungsbestimmungen des AEUV (vornehmlich Art. 145, 151 bis 158, 162 AEUV), aber auch dessen Bestimmungen, die gesellschaftliche Voraussetzungen und Folgen von Arbeit regeln (öffentliche Daseinsvorsorge, Umwelt, berufliche Bildung etc.) (Art. 14 und z.B. 168, Art. 191 ff., Art. 165 ff. AEUV). Hierzu gehören die durch Art. 6 Abs. 1 EUV mit dem Vertragsrecht für gleichrangig erklärten Bestimmungen der Charta der Grundrechte. ${ }^{33} \mathrm{Zu}$ berücksichtigen bei der Heranziehung diverser Rechtsquellen für eine europäische soziale Verfasstheit ist das primärrechtliche Kohärenzgebot, das Art. 7 AEUV für die gemeinschaftliche Zielverfolgung über ihre verschiedenen Aufgabengebiete hinweg formuliert. ${ }^{34}$

$\mathrm{Zu}$ den zitierten primärrechtlichen Bestimmungen gehören Art. 6 Abs. 2 und 3 EUV sowie Art. 151 AEUV. Diese verweisen ihrerseits auf internationale Dokumente, die in den Zielhorizont der EU einbezogen sind (Europäische Sozialcharta, Gemeinschaftscharta der sozialen Grundrechte der Arbeitnehmer von 1989, ${ }^{35}$ Europäische Konvention zum Schutz der Menschenrechte und Grundfreiheiten). Dabei kommt letzterer insofern eine besondere Rolle zu, als Art. 6 Abs. 2 EUV eine primärrechtliche Beitrittspflicht begründet. ${ }^{36}$ Diese internationalen Dokumente verweisen auf weitere, von allen MS getragene internationale Rechtsakte sozialer Dimension als Quelle (auch) einer europäischen Sozialstaatlichkeit - dazu gehören von allen MS getragene Abkommen und Empfehlungen der Internationalen Arbeitsorganisation (ILO), Konventionen der Vereinten Nationen (UN) (zu wirtschaftlichen, sozialen und kulturellen Rechten, zur Beseitigung jeder Form der Diskriminierung der Frauen, zur kulturellen Vielfalt, zum Umgang mit Behinderung etc.).

33 S. AB1. EU 2010 C 83/02.

34 Art. 7 AEUV lautet: „Die Union achtet auf die Kohärenz zwischen ihrer Politik und ihren Maßnahmen in den verschiedenen Bereichen und trägt dabei unter Einhaltung des Grundsatzes der begrenzten Einzelermächtigung ihren Zielen in ihrer Gesamtheit Rechnung." (Hervorhebung U.M.).

35 Art. 151 AEUV: ,... eingedenk der sozialen Grundrechte, wie sie in der am 18. Oktober 1961 in Turin unterzeichneten Europäischen Sozialcharta und in der Gemeinschaftscharta der sozialen Grundrechte der Arbeitnehmer von 1989 festgelegt sind, ...".

36 Dazu A. Hatje, in: Schwarze (Fn. 21) Art. 6 EUV, Rn. 10 ff.; ferner K. Lörcher, Zum Beitritt der Europäischen Union zur Europäischen Menschenrechtskonvention, Arbeit und Recht, 2013, S. 23-27. 
Speziell Art. 6 Abs. 3 EUV eröffnet darüber hinaus ein für die Analyse europäischrechtlicher Sozialstaatlichkeit neues Feld von Rechtsquellen. Er bezieht sich auf gemeinsame Verfassungstraditionen als Quelle (auch) europäischen Rechts. Dieser Rechtsquelle kommt umso größere Bedeutung zu, als die Rechtsprechung des $\mathrm{EuGH}^{37} \mathrm{zu}$ ihr zu einem extensiven Ergebnis gelangt ist. Danach sind diese gemeineuropäischen Verfassungstraditionen nicht als das numerische Mittelmaß der Grundrechte aller Mitgliedstaaten zu verstehen. Sie setzen also nicht eine vollständige Summe aller einzelstaatlichen Verfassungstraditionen voraus. Vielmehr können auch grundrechtliche Akzentuierungen einzelner MS, denen in anderen MS Entsprechungen fehlen, Bausteine solcher gemeineuropäischer Tradition sein. Anstelle eines „Maximalkatalogs“ (allgemeine Verbreitung einer Verfassungstradition) oder eines „Minimalkatalogs“ (ihre nur punktuelle Verbreitung) verwendet der EuGH heute den Maßstab der sog. ,"negative[n] ' Kontrollfunktion“: Er prüft, „ob eine konkrete Maßnahme mit dem Grundrechtsstandard eines Mitgliedstaats unvereinbar ist. Da bei der Beschränkung der Grundrechte auch der unionsrechtlichen Dimension angemessen Rechnung getragen werden muss (,relativierter Maximalstandard'), führt dieser Ansatz zu praktikablen Ergebnissen. "38

Diese Begründungsvariante für die Annahme gemeinsamer Verfassungstraditionen i.S.v. Art. 6 Abs. 3 EUV trägt dem europäischen Mehrebenen-System in besonderer Weise Rechnung. Ohne sich über nationale Regulierungsbefugnisse hinwegzusetzen, bringt sie diese doch zugleich in einen dynamischen Zusammenhang mit dem in der EU ,,vernetzten“"39 Staaten- und Werteverbund. Dieser Verbund ist zwar aus Diversität entstanden, gleichwohl aber auf wechselseitige Lernprozesse angelegt und lässt sich dabei von leitenden Zielen und Gemeinschaftstreue bewegen und entwickeln.

\section{Beziehungen von europäischer sozialer Verfasstheit zum „Europäischen Sozialmodell ${ }^{6}$}

Gesucht und postuliert wird in diesem Beitrag eine der EU inhärente Bindung an ein bisher nur nationalstaatlich interpretiertes Ziel der Sozialstaatlichkeit. Gesucht wird also nicht ein weiteres „Sozialmodell“ für Europa. ${ }^{40}$ Die in der Literatur vorgeschlagenen ,europäischen Sozialmodelle“ sind entweder deskriptiver Art - als Evaluationen und/oder Bewertungen dessen, was in Europa auf sozialem Gebiet

37 S. die Aufstellungen bei R. Streinz in: Streinz (Fn. 21), Art. 6 EUV Rn. 29-33; R. Geiger in: Geiger/Khan/Kotzur, EUV/AEUV-Kommentar, 2010, 5. Aufl., Art. 6 EUV, Rn. 33.

38 So R. Streinz in: Streinz (Fn. 21), Art. 6 EUV, Rn. 26.

39 A. v. Bogdandy (Fn. 7), Art. 6 Abs. 2, 3 EUV, S. 30.

40 S. entsprechende Hinweise bei A. v. Bogdandy (Fn. 7), S. 70 f.; E. Eichenhofer (Fn. 16); E. Eichenhofer, Das Europäische Sozialmodell, in: Deutscher Sozialrechtsverband (Hrsg.), Sozialrecht in Europa, 2010, S. 139-162; B. Bercusson, European Labour Law, 2009, 2. Aufl.; S. Huster (Fn. 9), S. 369-380 (der europäische Sozialstaatlichkeit auf S. 369 explizit ablehnt und auf S. 378/79 lediglich auf ein „europäisches Sozialmodell“ verweist); C. D. Classen in: Oppermann/Classen/Nettesheim (Fn. 22), S. 495; W. Weiß, Öffentliche Daseinsvorsorge und soziale Dienstleistungen: Europarechtliche Perspektiven, EuR 2013, S. 669-687 (hier S. 679-682). 
besteht und geschieht. Oder sie sind postulativer Art - als Statements, wie Europa (vom Blickwinkel des Autors/der Autorin aus betrachtet) sozial sein müsste. ,'Europäisches Sozialmodell“" - so schreibt denn auch Eberhard Eichenhofer - ,bezeichnet keinen Rechtsbegriff, sondern steht für ein politisches Konzept."41

Im vorliegenden Beitrag wird hingegen ein bereits geltender normativer Prüfungsmaßstab („Messlatte“) für soziale Regulierung gesucht, die sich die EU und ihre MS in den o.a. Rechtsquellen selbst gesetzt haben und an die damit die Mitglieder des Mehrebenen-Systems Europa sich selbst und die EU gebunden haben. Diese Messlatte sozialer Verfasstheit ist eine rechtlich verbindliche. Gewiss steht sie in praktischer Konkordanz mit anderen den obigen Rechtsquellen zu entnehmenden normativen Zielen. Aber sie ist nicht eine beliebige, neben anderen Zielen (wie Wirtschaftlichkeit, Effizienz, best practices etc.) stehende Variable einer wünschenswerten oder befürworteten Politik Europas. Das unterscheidet sie von beschriebenen oder postulierten ,europäischen Sozialmodellen“"

Die im Folgenden ausformulierte soziale Verfasstheit Europas stellt eine - zumindest objektivrechtlich ${ }^{42}$ - verbindliche Zielbestimmung dar. D.h. sie muss eingehalten werden, wenn nicht andere gleichrangige rechtliche Ziele ihr entgegenstehen und im Verhältnismäßigkeitsvergleich den Vorrang beanspruchen können. Dass sich eine so ausformulierte Sozialverfassung nicht dem Wandel entziehen kann, teilt sie mit allen Normen. Aber wollen die EU- und MS-Akteure von ihr abweichen, müssen sie diesen Maßstab sozialer Verfasstheit zunächst abschaffen oder modifizieren. Dann erst sind sie zu abweichenden Maßnahmen oder Normsetzungen befugt.

\section{Normative Gestalt der europäischen Sozialverfassung}

Nach den in Abschnitt IV. genannten Rechtsquellenüberlegungen muss der Ausgangspunkt der Bestimmung eines rechtlich verbindlichen europäischen Kriteriums der Sozialstaatlichkeit beim europäischen Primärrecht liegen. Dessen Prinzipien werden durch darin in Bezug genommene internationale Abkommen sowie durch gemeinsame Verfassungstraditionen der Mitgliedstaaten ergänzt und z.T. konkretisiert.

41 E. Eichenhofer (Fn. 16).

42 Objektivrechtlich meint, dass eine rechtliche Norm die Normadressaten zur Normeinhaltung verpflichtet. Die objektivrechtliche Verbindlichkeit kann unabhängig davon bestehen, ob sie Rechtssubjekten Ansprüche einräumt, die diese „subjektivrechtlich“ (z.B. im Wege der Klage) geltend machen können. Nur objektivrechtliche Normen sind selten - der Prototyp ist das Haushaltsgesetz. Vermutlich sind echte verfassungsmäßige Staatsziele „nur objektivrechtliche“ Normen. Sie können entweder nur in besonderen Verfahrensarten - wie dem Normenkontrollverfahren vor dem Bundesverfassungsgericht - oder nur in Verbindung mit subjektiven Rechten im allgemeinen Klageverfahren durchsetzt werden. 


\section{Sozialstaatlichkeit als Grundprinzip}

Im Folgenden wird die Annahme begründet, dass dem europäischen Recht - gemäß den o.g. Rechtsquellen - ein Prinzip der sozialstaatlichen Verfasstheit wenigstens auf dem Gebiet der abhängigen Arbeit innewohnt. Sozialstaatlichkeit wird im gegebenen Zusammenhang als das verstanden, was in den Arbeiten zum Europäischen Verfassungsrecht als „Prinzipien“, „Grundprinzipien“ u.ä. bezeichnet wird. Diese Prinzipien beziehen sich auf weitere Gebiete als das hier verfolgte Gebiet der Regulierung abhängiger Arbeit. Gleichwohl muss auch ein gebietsspezifischer Begriff von sozialer Verfasstheit in die allgemeine verfassungstheoretische und -rechtliche Debatte eingeordnet sein.

Armin v. Bogdandy verwendet das Wort „Grundprinzip“ als einen „rechtswissenschaftlichen" Begriff, um in der Tradition des Konstitutionalismus jene Normen des Primärrechts zu identifizieren und zu deuten, die eine normativ begründende Funktion für das Ganze der Unionsrechtsordnung haben; sie legen die angesichts der Rechtfertigungsbedürftigkeit hoheitlichen Handelns wesentlichen legitimatorischen Grundlagen fest. ${ }^{43}$ Christian Calliess spricht von zu Strukturmerkmalen der EU verdichteten werthaltigen Prinzipien. Unter dem Titel „Wertbegriff und Europäischer Werteverbund“ stellt er fest: „In diesem Kontext vermitteln Werte freilich nicht nur die Legitimation, sondern sie können - vermittelt über ihren Prinzipiengehalt - als Auslegungsleitlinie und Normenkontrollmaßstab dienen. ...". Diese Prinzipien seien zu „Strukturmerkmalen der EU“ geworden. ${ }^{44}$ Bei ihnen handelt es sich um normative Strukturmerkmale, die i.A. im Verfassungsrecht niedergelegt sind. Sie legitimieren - bzw. im negativen Falle: delegitimieren - in doppelter Weise. Einmal begründen sie rechtlich die Herrschaft sozialer Akteure. Zum anderen gewährleisten sie die interpretierende Kontrolle ihrer Einhaltung durch diese Akteure.

\section{Auf Arbeit bezogene europäische Sozialverfassung im Primärrecht}

Die Annahme, dass dem europäischen Recht - gemäß den o.g. Rechtquellen - ein Prinzip der sozialstaatlichen Verfasstheit auf dem Gebiet der abhängigen Arbeit innewohnt, ${ }^{45}$ wird im Folgenden (unten 3.) gestützt auf die verfassungsmäßigen Werte der Menschenwürde, der Demokratie und ergänzend der Solidarität. Diese

43 A. v. Bogdandy (Fn. 7), S. 27. Dem hier interessierenden Gebiet näher steht der Begriff einer arbeitsverfassungsrechtlichen „Leitnorm“ bei $F$. Rödl (Fn. 12), S. 872. Diese umschreibt ,,alle diejenigen Verfassungsnormen ..., die jenseits der konstitutiven Grundrechte der sozialen Akteure juridische Effekte im gesellschaftlichen Feld abhängiger Arbeit entfalten können“. Auch Rödl sucht die dem deutschen Prinzip der Sozialstaatlichkeit (Art. 20 Abs. $1 \mathrm{GG}$ ) entsprechende europäische Leitnorm (S. 860).

44 C. Calliess in: Calliess/Ruffert (Fn. 21).

45 Interessanterweise wird diese Annahme tendenziell eher von Autoren geteilt, die sich europäischem Arbeitsund/oder Sozialrecht zuwenden: Bahnbrechend etwa H. F. Zacher, Wird es einen europäischen Sozialstaat geben?, in: EuR, 2002, S. 147 ff. Demgegenüber sind Autoren des europäischen Verfassungsrechts eher skeptisch, oder gehen auf potentielle Sozialstaatlichkeit der EU gar nicht ein - wie T. Giegerich, Europäische Verfassung und deutsche Verfassung im transnationalen Konstitutionalisierungsprozess, 2003. 
Werte haben im Primärrecht zunehmende Bedeutung erlangt und sowohl im Primärrecht als auch im Sekundärrecht, den im Primärrecht bezogenen internationalen Abkommen wie auch in den gemeinsamen Verfassungstraditionen der Mitgliedstaaten eine konkretisierende Verstärkung erfahren.

Das europäische Primärrecht begann in seinem Entwicklungsprozess mit der Anerkennung nur ökonomischer Integrationsziele. Erst allmählich - v.a. mit der Einheitlichen Europäischen Akte, dem Maastricht-Vertrag und dem Sozialpolitik-Protokoll, dem Amsterdamer Vertrag sowie dem Lissaboner Vertrag - gab es der sozialen Integration eine eigene rechtliche Kontur. ${ }^{46}$ Uneinigkeit besteht darin, ob man heute bereits von einer „Balance“ zwischen Ökonomischem und Sozialem sprechen kann $^{47}$ und inwieweit diese Balance durchsetzungsfähig und stabil ist. Rebhahn/ Reiner konstatieren: „Der sukzessive Ausbau sozialer Kompetenzen und Zielsetzungen brachte zwar eine bessere Basis für eine ausgewogene Abwägung sozialer und wirtschaftlicher Interessen. Allerdings können , harte Regeln“ eher durchgesetzt werden als ,weiche Ziele“". 48 Und Paul Craig stellt die Aussagekraft von Primärrecht in dieser Hinsicht gänzlich in Frage, wenn er schreibt: „The ,true balance' between the economic and the social at any stage of the Community's existence can only be divined in part by perusal of changes to the primary Treaty articles. Three factors have always been crucial in this respect, and will remain so in the post-Lisbon world. The nature and content of EU legislation made pursuant to these powers, their interpretation by the Union courts, and the priority accorded to different aspects of Union policy by the key institutional players will continue to shape the reality of the divide between the economic and the social as it has done in the past."49

Ein dem deutschen Grundgesetz vergleichbares Sozialstaatsprinzip hat sich im europäischen Primärrecht bislang expressis verbis nicht niedergeschlagen. Wohl aber wurde im Lissaboner Abkommen, Art. 3 Abs. 3 EUV, zum ersten Mal in der Geschichte der EU der Begriff der „,sozialen Marktwirtschaft“ verankert. Im deutschen Recht wird der Begriff der sozialen Marktwirtschaft als die unter dem Vorbehalt des Sozialstaatsprinzips stehende Wirtschaftsordnung der Marktwirtschaft verstanden. Jedoch ist diese Interpretation nicht unbestritten. ${ }^{50}$ Sie kann auch nicht o.w. (selbst wenn die englische und französische Fassung ,social market economy“ und „économie sociale de marché" der deutschen entsprechen) einem europäischen Dokument unterlegt werden. Gleichwohl stellt Art. 3 Abs. 3 EUV entgegen anderslau-

46 So auch F. Mayer und die Mehrzahl der in der von der Friedrich-Ebert-Stiftung 2009 veröffentlichten Kurzbeiträge zu seiner gutachtlichen Stellungnahme (siehe Mayer (Fn. 6), S. 7-25) und die Kurzbeiträge in der gleichnamigen Schrift der FES 2009 Friedrich-Ebert Stiftung (Fn. 6).

47 Zweifelnd D. Grimm, Die Zukunft der Verfassung II: Auswirkungen von Europäisierung und Globalisierung, 2012.

48 S. Krebber in: Calliess/Ruffert (Fn. 21), Art. 151 AEUV, Rn. 9.

49 P. Craig, The Lisbon Treaty. Law, Politics, and Treaty Reform, 2013, S. 329.

50 K. A. Schachtschneider (Fn. 32) erklärt bereits für Deutschland den Begriff der sozialen Marktwirtschaft als mit dem menschheitsrechtlich begründeten Sozialprinzip unvereinbar. Er wirft der durch Lissabon geformten europäischen Wirtschaftsverfassung - insb. Art. 3 und 6 EUV - vor, „der marktlichen Sozialwirtschaft, der Wirtschaftsverfassung Deutschlands, keine Chance (zu lassen).“. 
tenden Positionen ${ }^{51}$ durchaus einen ersten Schritt zu einer sozialverfassungsrechtlichen Charakterisierung der europäischen Wirtschaftsordnung dar. Die Bestimmung ist zumindest ein Indiz für die rechtliche Anerkennung einer europäischen Sozialstaatlichkeit.

\section{Menschenwürde, Demokratie und Solidarität als Quellprinzipien}

$\mathrm{Zu}$ einem Geltungsgrund für ein Sozialstaatsprinzip im europäischen Primärrecht wird dieses Indiz, wenn man es in Beziehung zu den drei, im folgenden abgehandelten, europäischen Verfassungswerten Menschenwürde, Demokratie und Solidarität setzt. Alle drei Werte haben für die europäische Verfassung und die europäische Identität in den letzten Jahrzehnten unstreitig erhöhte - sogar zentrale - Bedeutung erlangt. Allen drei Werten, so wird zu zeigen sein, wohnt neben subjektivrechtlichen eine objektivrechtliche soziale Staatszielkomponente inne, die sich auf einen gebotenen staatlichen Umgang mit der sozialen Frage oder Bestandteilen von ihr beziehen. V.A. Menschenwürde und Demokratie stellen Quellprinzipien dar, die sich nicht in dem formulierten Recht oder der etablierten Institution an sich schon erschöpfen, sondern die darüber hinaus auf andere Rechte/Rechtsgebiete oder Institutionen ausstrahlen. Beide geben gerade für das hier beobachtete Rechtsgebiet der abhängigen Arbeit zentrale normative (Interpretations- und Regulierungs-) Parameter ab. Sie stellen daher für die arbeitsbezogene europäische Sozialverfassung einen positiven Geltungsgrund dar.

51 M. Pechstein, in: Streinz (Fn. 21), schreibt zu Art. 3 Abs. 3 EUV zwar, er sei „rechtsverbindlich“, nicht nur Programmsatz (Rn. 3). Er setzt dann freilich ohne jede Begründung hinzu: „Neu, wenngleich ohne eigenständige Bedeutung, ist das ausdrückliche Bekenntnis zu einer sozialen Marktwirtschaft" (Rn. 7). Entsprechend enthält die Kommentierung von Abs. 3 und 5 dem Leser jede juristisch solide Erläuterung vor. Auch M. Ruffert, in: Calliess/Ruffert (Fn. 21), Kommentierung zu Art. 3 hält Art. 3 zugute, er konstituiere ein verbindliches (wenn auch „nur in sehr engen Grenzen justitiables“) und wohl exklusives Zielbündel (Rn. 5 und 6). Hinsicht der wettbewerbsfähigen sozialen Marktwirtschaft setzt M. Ruffert aber gleichfalls ohne juristische Argumente hinzu: „muss sich die verfassungsrechtliche Wirkung dieser Änderung auf eine semantische beschränken lassen“ (Rn. 25). „Das Europäische ,Sozialmodell“ soll sich von sozioökonomischen Strukturen der USA abheben“ (Rn. 26). Daher sei mit Art. 3 Abs. 3 EUV keine „Systementscheidung“ i. S. d. deutschen Tradition der sozialen Marktwirtschaft verbunden (Rn. 38 - auch unter Verweis auf Art. 345 AEUV). - Erstaunlich ist, wie beide Autoren gleichzeitig die Neuheit und Rechtsverbindlichkeit der neuen Charakterisierung der Wirtschaftsordnung Europas konstatieren $u n d$ ohne jedes schlüssige Argument dieser Rechtsverbindlichkeit durch Ignorierung die Gefolgschaft verweigern. Sie bieten damit ein gutes praktisches Anwendungsbeispiel für die skeptische Prognose von P. Craig (Fn. 49), ,the priority accorded to different aspects of Union policy by the key institutional players will continue to shape the reality of the divide between the economic and the social." $W$. Wei $\beta$ (Fn. 40) gelangt in seiner Analyse des Begriffes der "sozialen Marktwirtschaft" zu dem Resultat von mit "sozialer Abfederung und sozialstaatlicher Begleitung funktionierende(n) Wettbewerbsprozesse(n)" (S. 681/682). Sicher ungewollt erfährt damit das Soziale eine gegenüber dem Wettbewerblichen abgeschwächte ("Abfederung", "Begleitung", nicht etwa "Balance" oder gar "Korrektur") rechtliche Bedeutung. Von einem durch die Aufnahme der sozialen Marktwirtschaft in das Primärrecht ungebrochenen "Regel-Ausnahme-Verhältnis" zwischen Marktlichem und sozialstaatlicher Intervention spricht denn auch C. Nowak (Fn. 11), S. 38 Rn. 85. Soziale Marktwirtschaft wird so zu einem bloßen Epipethon ("Akzentverschiebung", "begrenzte Rechtsfolgen im Übrigen") (S. 38) und läuft rechtlich leer. 


\section{a) Menschenwürde}

Auf den rasanten und zugleich expansiven Aufschwung der „Menschenwürde“ im europäischen Wertesystem hat Christian Calliess ${ }^{52}$ aufmerksam gemacht. In seiner Darstellung des „Wertbegriffs und des Europäischen Werteverbunds“ reiht er die Menschenwürde unter die Prinzipien ein, die zu „Strukturmerkmalen der EU“ geworden sind. Menschenwürde sei als „Schlüssel-Wert bzw. Quellcode der Architektur des europäischen Staats- und Verfassungsverbunds, des verfassungsrechtlichen Wertesystems schlechthin zu verstehen“. Ihr herausgehobener Charakter ergebe sich auch aus der Stellung in Art. 1 der Charta der Grundrechte der Europäischen Union (GRCh), „dem eigentlichen Fundament der Grundrechte“, womit sie „gleichzeitig auch echtes Grundrecht“" sei. Auch in der neueren Rechtsprechung des EuGH sei Menschenwürde ,als objektives Verfassungsprinzip und Grundrecht der Union und damit als Kontrollmaßstab europäischer Gesetzgebung anerkannt" ${ }^{\text {"5 }} 33$

Menschenwürde stellt sich im deutschen wie im europäischen Verständnis ${ }^{54}$ als allgemeine und der Entwicklungsdynamik gegenüber offene Norm dar. An einer verbindlichen normativen Aussage aber hat sie unbestritten teil: Der Mensch darf nicht zum bloßen Objekt des Handelns anderer gemacht werden. „Aufgrund und wegen seiner Würde darf der Mensch niemals bloßes Mittel sein, um einen Zweck zu erreichen. "55 Diese allgemeine prinzipielle Bedeutung von Menschenwürde nach Art. $1 \mathrm{GRCh}$ hat drei Komponenten. Die freiheitliche Komponente gebietet den Schutz von Individualität, Identität und Integrität des Menschen. Die egalitäre Komponente hingegen garantiert Schutz vor Verletzung des Gleichheitsgedanken. Und die dritte - soziale - Komponente gebietet die Gewährleistung sozialer Rechte und Grundsätze. ${ }^{56}$

Die Ausstrahlung des Grundwerts der Menschenwürde auf das Gebiet abhängiger Arbeit ist unmittelbar plausibel. Alle drei genannten Komponenten finden auf dem Gebiet von Arbeit besondere Bedingungen vor. Der Identitäts- und Integritätsschutz ist durch die dauerhafte Eingliederung in die fremde Herrschaftssphäre Betrieb herausgefordert. Der Gleichheitsschutz wird durch einseitige Auswahl- und Direktionsbefugnis des Arbeitgebers heraufbeschworen. Und sozialer Schutz wird v.a. durch die kontinuierliche Unterworfenheit von Arbeitnehmern unter fremde Weisung notwendig. Sicherlich werden diese drei Spannungsverhältnisse konkret und im Detail unterschiedlich im jeweiligen MS perzipiert und rechtlich gelöst. Aber alle drei zeigen an, dass die Gebote der Menschenwürde in das Feld abhängiger

52 C. Calliess, in: Calliess/Ruffert (Fn. 21), Art. 2 EUV, Rn. 16 f.

53 Ähnlich J. Schwarze, in: Schwarze (Fn. 21), Art. 2 EUV, „Bemerkenswert ist, dass die Menschenwürde an vorderster Stelle genannt ist.“ (Rn. 1) Art. 2 sei verbindlich, zwar allgemein, aber er gebe „Orientierungspunkte für die Auslegung“ (Rn. 2). Noch weiter - zumindest geographisch - geht P. Häberle (Fn. 7), S. 547, „Weltweit werden so Minimalstandards der Menschenwürde, Sozial- und Umweltverträglichkeit erforderlich".

54 Dazu bereits vor Inkrafttreten des Lissaboner Vertrages die Länder-Übersicht bei M. Borowski in: Meyer (Hrsg.) Charta der Grundrechte der Europäischen Union, 2011, 3. Aufl., Art. 1 GRC Rn. 2-5.

55 M. Borowski, in: Meyer (Fn. 54).

56 M. Borowski, in: Meyer (Fn. 54), Art. 1, Rn. 6-25. 
Arbeit hineinreichen und dort Regulierungsbedarfe aufwerfen. Dass Menschenwürde rechtlich ,gilt" und nicht nur ein programmatisches Epitheton darstellt, hat unmittelbar Folgen für die Regulierung von Arbeit. Menschenwürde muss daher als erster und prinzipiellster Bestandteil europäischer Sozialverfassung betrachtet werden.

\section{b) Demokratie}

Wie Menschenwürde hat auch Demokratie in den letzten Jahrzehnten einen sprunghaften Aufschwung unter den Grundwerten der EU erfahren. Aufgrund dieses Bedeutungszuwachses strahlt denn auch der Wertgehalt der Demokratie in andere Regelungsgebiete aus. ${ }^{57}$ Die Römischen Verträge von 1957/58 waren weit davon entfernt, den normativen Wert der Demokratie auf die damals als rein wirtschaftliche Gemeinschaft verstandene Europäische Wirtschaftsgemeinschaft (EWG) zu beziehen. Demgegenüber haben die Bildung des Europäischen Parlaments (EP), dessen Direktwahl seit 1979 sowie die fortwährende Ausdehnung von dessen Einfluss gegenüber dem Ministerrat dem Demokratiegedanken zum Durchbruch verholfen und ihm innerhalb des europäischen Verfassungsrechts immer höher angesiedelt. Demokratie ist, wie sich v.A. am Lissaboner Vertrag zeigen lässt, zu einem normativen Kernmerkmal europäischer Identität geworden.

Wie im Falle der Menschenwürde schlägt sich dieser primärrechtliche Bedeutungszuwachs auch in der veränderten Architektur des Lissaboner Vertrages nieder. Art. 2 EUV reiht Demokratie noch (wie zuvor) in den Katalog der obersten Werte mit „Achtung der Menschenwürde, Freiheit, Demokratie, Gleichheit, Rechtsstaatlichkeit und die Wahrung der Menschenrechte [neu] einschließlich der Rechte der Personen, die Minderheiten angehören," ein. Neu ist am Vertrag von Lissabon historisch gesehen ein Erbe des gescheiterten Verfassungsvertrages -, dass den allgemeinen Bestimmungen ein neuer ,Titel II. Bestimmungen über die demokratischen Grundsätze" folgt. Die darin enthaltenen Art. 10 und 11 EUV nehmen eine zweifache Um- und Aufwertung des Demokratischen im europäischen Verfassungsrecht vor. Erstens löst sich Art. 10 von der Vorstellung, Demokratie sei wesentlich ein Wert innerhalb der Mitgliedstaaten - und nur darüber vermittelt ein Wert der EU. Die EU selbst wird vielmehr dem Demokratiegebot unterstellt, indem Art. 10 Abs. 1 formuliert: „Die Arbeitsweise der Union beruht auf der repräsentativen Demokratie.“ (Hervorhebung von mir, U.M.) Zum Anderen erweitern beide Artikel den Wert der Demokratie über das Wahl- und Institutionsgeschehen hinaus und öffnen ihn zu unmittelbarer Bürgerbeteiligung. Art. 10 Abs. 3 bekräftigt: ,Alle Bürgerinnen und Bürger haben das Recht, am demokratischen Leben der Union teilzunehmen. Die Entscheidungen werden so offen und bürgernah wie möglich getroffen.“ Art. 11 öffnet den europäischen politischen Prozess für die Beteiligung

57 Während Menschenwürde einen substantiellen Wert verkörpert, ist der Wertgehalt von Demokratie von wesentlich prozeduraler Natur. 
der europäischen Zivilgesellschaft - bis hin zur Einrichtung der Europäischen Bürgerinitiative, die folgerichtig in Abs. 4 verankert ist.

Der Wert des Demokratischen besteht wesentlich darin, dass Normsetzung im politischen Gemeinwesen selbstbestimmt stattfindet. Er verlangt, dass Normbetroffene im Prinzip auch Normsetzer sein könnten ${ }^{58}$ - dass ein Nexus besteht bzw. hergestellt wird zwischen der Stimme (voice) und den Berechtigungen (entitlements) derer, von denen alle Staatsgewalt ausgeht. ${ }^{59}$ Diesem demokratischen Prinzip hat sich die EU über Krisen und Legitimationseinbrüche hinweg deutlich genähert und besonders markant sich im Abkommen von Lissabon darauf verpflichtet.

Im vorliegenden Zusammenhang interessieren v.a. die Ausstrahlung und der Einfluss des Demokratieprinzips auf die europäische soziale Verfasstheit. Der Durchbruch des Demokratischen als höchster Wert im EU-Verfassungsgefüge strahlt in doppelter Weise auf das Feld der Verfassung abhängiger Arbeit aus. Er verfasst das Feld der Normgenese (wie Normen produziert werden müssen, um als demokratisch legitim anerkannt werden zu können) und das Feld der Norminhalte (wie Normen inhaltlich beschaffen sein müssen, um als demokratisch legitim anzuerkennen zu sein).

Erstens: Normgenetisch steht soziale Regulierung arbeitsbezogener Art nationalstaatlich wie europäisch in einem Spannungsverhältnis zum „Selbstlauf“ der Arbeitsmarktprozesse (s. bereits oben Abschn. II. 1.). Sie sichert und korrigiert Marktprozesse außermarktlich aus Gründen gesellschaftlicher Nachhaltigkeit und Gerechtigkeit. Diese außermarktliche Kontrolle und Korrektur von Marktprozessen ist eo ipso keine ökonomische, sondern eine politische: Sie geschieht durch demokratische Eingriffe in das Marktgeschehen. Da es dabei immer um die Schlichtung von Konflikten zwischen partikularen Perzeptionen, Interessen- und Machtlagen geht, ist das Verhältnis von kapitalistischem Markt und Demokratie von Beginn des neuzeitlichen Arbeitsrechts an ein konfliktreiches. ${ }^{60}$ Unlängst hat dies Peter Häberle in seiner Europäischen Verfassungslehre erneut verdeutlicht. ${ }^{61}$ Markt und Demokratie - so Häberle - können nur koexistieren bei Vorliegen ,kräftiger Rahmenbedingungen (besser Konstitutionsbedingungen), ja rechtlicher Kontrollen und nicht

58 J. Habermas, Faktizität und Geltung. Beiträge zur Diskurstheorie des Rechts und des demokratischen Rechtsstaates, 1992.

59 U. Mückenberger, Civilising Globalism: Transnational Norm-Building Networks as a Lever of the Emerging Global Legal Order, in: Transnational Legal Theory, 2010, S. 523-573; U. Mückenberger/C. Jakobeit/R. Kappel, Zivilisierung der Weltordnung. Normbildung durch transnationale Netzwerke, in: Leviathan, 2010, S. 411-427.

60 Diese Konfliktgeschichte reicht in die Zeit der ersten deutschen Demokratie zurück. S. bereits H. Sinzheimer, Arbeitsrecht und Rechtssoziologie, darin insb.: Die Demokratie des Arbeitsverhältnisses, 1976, 2. Bd., S. 115-134; T. Ramm (Hrsg.), Arbeitsrecht und Politik: Quellentexte 1918-1933, 1966; J. Perels, Kapitalismus und politische Demokratie. Privatrechtssystem und Gesellschaftsstruktur in der Weimarer Republik, 1973, bis hin zu W. Streeck, Gekaufte Zeit. Die vertagte Krise des demokratischen Kapitalismus, 2013. Den grundlegenden Zusammenhang zwischen Demokratie und Sozialstaat unterstreicht auch F. Rödl, Zu Begriff und Perspektiven demokratischer und sozialer Union, in: Bast/Rödl (Fn. 11), S. 179-204, der ebendeshalb lediglich zum Befund eines "im Vergleich mit dem demokratischen Verfassungsstaat zu konstatierenden sozialstaatlichen Defizit(s) der Union" gelangt (S. 192). Ich teile diesen Befund als empirischen, nicht aber - wie dieser Beitrag zu belegen versucht - als normativen.

61 P. Häberle (Fn. 7), S. 544-561. Dort finden sich auch bereits weitblickende Anmerkungen zu einer erforderlichen europäischen Sozialstaatlichkeit. 
weniger sozialstaatlicher ,Eingriffe ‘ im Rahmen der Gerechtigkeit, z.B. zum Schutz der Schwächeren."62 Häberle kommt darüber zur Bestätigung der schon genannten Innovation des Lissaboner Vertrages: „Die soziale Marktwirtschaft ist verfassungsimmanentes Prinzip des Typus ,Verfassungsstaat' geworden“. Er gelangt darüber zu der Aussage (bereits zitiert): „Weltweit werden so Minimalstandards der Menschenwürde, Sozial- und Umweltverträglichkeit erforderlich.“ Für den Fortgang der Überlegungen ist Häberles Schlussfolgerung wichtig: „,Die in Gestalt der Tarifautonomie gefundene, stets neu gefährdete Balance der Arbeitgeber- und Arbeitnehmerinteressen bildet dabei ein Fundament seiner ,sozialen' Marktwirtschaft". ${ }^{63}$ Soziale Regulierung auf dem Arbeitsgebiet setzt somit schon normgenetisch Demokratie voraus.

Damit ist - zweitens - die norminhaltliche Ausstrahlung des Demokratischen auf das Feld der Verfassung abhängiger Arbeit in Europa angesprochen. Demokratie erfordert einen voice-entitlement-Nexus abhängig Beschäftigter im Arbeitsleben. Dazu muss ihre Stimme (voice) aus der Vereinzelung befreit und mit der realen Chance zur Bildung und Betätigung von Koalitionen und Interessenvertretungen ausgestattet werden. Koalitionsbetätigung und daraus hervorgehende Normbildung erhöhen - wie wir aus der Arbeits- und Sozialgeschichte wissen - die Legitimität und die Effektivität sozialer Normgebung im Vergleich zu nur staatlichem, auch supranational staatlichem Handeln. Staatliches Handeln dient hier deshalb nicht im Regelfall unmittelbar der Setzung substantieller Standards. Es dient auch und vorrangig der Herstellung prozeduraler Vorkehrungen zur Selbstregulierungsfähigkeit durch die sozial Betroffenen selber. Damit sollen - was auf norminhaltlicher Ebene der normgenetischen Komponente des Demokratieprinzips entspricht - sachnähere Akteure (Sozialpartner, Soziale Dialoge) befähigt und in die Lage versetzt werden, solche substantiellen Standards selbst zu regeln und durchzusetzen.

Die inhaltliche Ausstrahlung des Demokratieprinzips auf das Feld abhängiger Arbeit folgt daher dem, was in der neueren Diskussion das Prinzip der horizontalen Subsidiarität genannt wird. ${ }^{64}$ Dieses Prinzip besteht nicht in staatlicher Inaktivität (laissez-faire und/oder legal abstentionism). Es besteht vielmehr darin, dass staatliche Aktivität normativ in zweierlei Weise geformt wird. Erstens sollen staatliche Instanzen auf derselben Ebene ${ }^{65}$ aktiv zu empowerment und zur Befähigung (capa-

Siehe P. Häberle (Fn. 7), S. 545, Hinweis auf den fast gleichlautenden Art. 45 Abs. 4 Nr. 1 der Verfassung Irlands.

63 P. Häberle (Fn. 7), S. 546-548.

64 B. Bercusson (Fn. 40), S. 526-528.

65 Daher horizontale Subsidiarität - diese steht in Gegensatz zu vertikaler Subsidiarität, die sich auf das Verhältnis der EU zu den Mitgliedstaaten bezieht. Zum Begriff s. European industrial relations dictionary, 2011, Stichwort: horizontal subsidiarity, Eurofound, Dublin (letztes Update 2011 - Download 21.07.13). Zur Geschichte s. die Interinstitutionelle Vereinbarung vom 25. Oktober 1993 zwischen dem Europäischen Parlament, dem Rat und der Kommission über die Verfahren zur Anwendung des Subsidiaritätsprinzips (soweit ersichtlich unveröffentlicht - in Bezug genommen in: Protokoll Nr. 30 zum Amsterdamer Vertrag 1997 über die Anwendung der Grundsätze der Subsidiarität und der Verhältnismäßigkeit) und das Dokument der Kommission KOM (93) 600 endg., Brüssel, 14. Dezember 1993, unter 6(c): Dort ist die Rede von der „recognition of a dual form of subsidiarity in the social field: on the one hand, subsidiarity regarding regulation at national and Community level; 
bilities) der unmittelbar beteiligten Akteure zu selbstbestimmter Normbildung beitragen. Zweitens sollen sie den unmittelbar beteiligten Akteuren im Rahmen staatlich gesetzter Regelwerke (wie Kollektiv- oder Tarifvertragsgesetzen) diese Normbildung in Eigenverantwortung überlassen. Dies meint proaktive Subsidiarität. ${ }^{66}$ Mit dem Demokratieprinzip ist somit ein zweiter, zunehmende Bedeutung entfaltender Bestandteil der auf Arbeit bezogenen europäischen Sozialverfassung aufgewiesen.

\section{c) Solidarität}

Neben Menschenwürde und Demokratie als Grundwerten der EU-Verfassung, die dem Prinzip europäischer sozialstaatlicher Verfasstheit Geltungsgrund verschaffen, ist zuletzt ,Solidarität" hinzugetreten. ${ }^{67}$ Solidarität ist neu verankert in Art. 2 und 3 EUV, konkretisiert für Notsituationen findet sie sich in Art. 222 AEUV. Für Armin v. Bogdandy stellt dieses Prinzip eine Säule des ,neuen, globalisierungstauglichen europäischen Sozialmodells“ dar. ${ }^{68}$ Für Bergé/Robin-Olivier ist sie eines der ,'principes généraux de droit' (ou sous-principes) qui se sont révélés necessaires à l'élaboration et à la mise en oeuvre des règles européennes. "69 Solidarität ist nicht in Art. 2 S. 1 EUV unter den ,Werte(n), auf die sich die Union gründet“" aufgeführt. Aber sie wird in S. 2 ,einer Gesellschaft“ (mit der angesichts des Singulars nur die gemeinsame europäische Gesellschaft gemeint sein kann), die sich u.a. durch Solidarität ,,auszeichnet"“, zugeschrieben und damit zu einem Leitwert der EU-Verfassung erklärt. ${ }^{70}$

on the other, subsidiarity as regards the choice, at Community level, between the legislative approach and the agreement-based approach." (Dok. unveröffentlicht - hier zitiert nach B. Bercusson (Fn. 40), S. 526).

66 Im Protokoll Nr. 2 über die Anwendung der Grundsätze der Subsidiarität und der Verhältnismäßigkeit des Lissaboner Vertrages finden sich Regelungen der horizontalen Subsidiarität nur noch in Erwägungsgrund 1 (,,in dem Wunsch sicherzustellen, dass die Entscheidungen in der Union so bürgernah wie möglich getroffen werden,“) und in Art. 2 (,Die Kommission führt umfangreiche Anhörungen durch, bevor sie einen Gesetzgebungsakt vorschlägt. Dabei ist gegebenenfalls der regionalen und lokalen Bedeutung der in Betracht gezogenen Maßnahmen Rechnung zu tragen.") Der Großteil der Regelungen dieses neuen Protokolls ist ausschließlich von der Sorge nationaler staatlicher Instanzen vor Souveränitätspreisgabe geleitet. Unstreitig unterliegt horizontale Subsidiarität nicht den Regeln, die Art. 5 Abs. 3 EUV (und Vorgängervorschriften) für vertikale Subsidiarität setzt: B. Bercusson (Fn. 40); Eurofound (Fn. 66), 2011. Welche Regeln stattdessen gelten, ,remain(s) to be elaborated“ - so B. Bercusson (Fn. 40), S. 528. Die beiden Leitgedanken zu proaktiver Subsidiarität im Text (1. Vorrang nichtstaatlicher autonomer substantieller Regulierung - 2. aber staatliche Verpflichtung, nichtstaatliche Akteure prozedural zu substantieller Regulierung zu befähigen) könnten Grundstein einer solchen Ausarbeitung werden.

67 Auf den Wert Solidarität beziehen sich A. v. Bogdandy (Fn. 7), S. 13-71; F. Rödl (Fn. 12); K. A. Schachtschneider (Fn. 32), S. $183 \mathrm{ff}$.

68 A. v. Bogdandy (Fn. 7), S. 70 f.

69 J. S. Bergé/S. Robin-Olivier, Droit européen. Union européenne, Conseil de l'Europe, 2011, 2. Aufl., S. 350 f.

70 So auch C. Calliess, in: Calliess/Ruffert (Fn. 21), Art. 2 EUV Rn. 30. Solidarität sei zwar nicht als Wert angeführt, sei aber gleichwertig (auch im Hinblick auf die Solidaritätsklausel in Art. 222 AEUV) und damit ein „Leitwert und Rechtsprinzip im europäischen Staaten-, Verfassungs- u Werteverbund“. 
Solidarität taucht häufig, aber keineswegs ausschließlich, als Verhaltensregel zwischen den Mitgliedstaaten auf (Art. 3 Abs. 3 UAbs. 3 EUV). ${ }^{71}$ Schon Art. 3 Abs. 3 UAbs. 2 EUV erweitert sie auf das Verhältnis zwischen Generationen und platziert sie unter weitreichenden sozialen Rechten. Auch insoweit nahm die GRCh - erst in der Form des nicht verbindlichen Nizza-Vorgängers, dann in der primärrechtsgleichen des Lissaboner Vertrages - eine deutliche normative Aufwertung vor. Schon die Präambel reiht Solidarität - neben Würde des Menschen, Freiheit und Gleichheit - in die Liste der „unteilbaren und universellen Werte“ ein. Weiter heißt es in der Präambel: „Die Ausübung dieser Rechte ist mit Verantwortung und mit Pflichten sowohl gegenüber den Mitmenschen als auch gegenüber der menschlichen Gemeinschaft und zukünftigen Generationen verbunden." Schließlich erhält Titel IV GRCh, der in Art. 27-38 wesentliche kollektive und individuelle arbeitsbezogene Grundrechte, aber auch Dienstleistungen von allgemeinem öffentlichen Interesse sowie Umwelt- und Verbraucherschutzpflichten enthält, die hervorhebende Überschrift ,Solidarität“.

Solidarität ist von zentraler Bedeutung für das Feld arbeitsbezogener Regulierung. Sie verweist für den Bereich des Arbeitslebens auf bewusste gesellschaftlich gestaltete Befriedigung von Bedürfnissen, und auf den Weg, wie zu dieser Bedürfnisbefriedigung zu gelangen ist. ${ }^{72}$ Darin liegen zwei normative Bestandteile.

Solidarität ist das aus der Gleichheit der Lebenslage Arbeitender folgende Verhältnis von Konkurrenz und Überwindung der Konkurrenz durch gemeinsame Organisation und gemeinsames Handeln. Damit ist sie der Ursprung von Koalitionen, gemeinsamer Aushandlung von Arbeitsbedingungen, notfalls Kampfmaßnahmen seitens abhängig Beschäftigter sowie von deren rechtlicher Anerkennung als Koalitionsfreiheit, Tarifautonomie und Arbeitskampfrecht. Diese Rechte sind denn auch

71 Die Einschränkung von Solidarität auf das Verhältnis zwischen EU-Mitgliedstaaten wird m.E. übergewichtet von A. v. Bogdandy (Fn. 7) und ihm folgend von F. Rödl (Fn. 12). Demgegenüber betont M. Rossi die Entwicklung von Solidarität ,zum vieldeutig verwendeten Rechtsbegriff im Recht der Europäischen Union“ (in: Calliess/ Ruffert, Fn. 21, Nr. 2 zu Art. 80 AEUV). Ebenso hebt C. Calliess darauf ab, dass die „Solidaritätspflicht“ „als eines der bestimmenden Leitmotive des unionalen Rechts gleich an mehreren Stellen in den Verträgen normiert" sei: Die neue Europäische Union nach dem Vertrag von Lissabon, Tübingen: Mohr-Siebeck 2010, S. 104. Meyer (Fn. 54, Nr. 19 zur Präambel) spricht vom Grundsatz der Solidarität als einer „,zusätzliche(n) Werteentscheidung“ - „neben der Freiheit, der Demokratie, der Achtung der Menschenrechte und der Rechtsstaatlichkeit“; ähnlich R. Streinz (Fn. 21), Nr. 1 vor Art. 27 GR-Charta. Auch U. Haltern (in: v. Bogdandy/Bast (Fn. 7) S. 279 ff., hier bei Fn. 148) behauptet zwar, das Solidarband sei im Vertrag in erster Linie auf das Verhältnis der Mitgliedstaaten untereinander bezogen“, sei aber vom EuGH ,auf das Verhältnis zwischen Individuen“ erstreckt worden.

72 C. Calliess spricht von zwei Erscheinungsformen: „Einerseits im Verhältnis des einzelnen gegenüber dem ganzen als ,Vorrang des gemeinsamen Interesses vor dem eigenen Interesse' und andererseits als Solidarität aller bzw. des ganzen gegenüber einzelnen in Hilfeleistungen gegenüber Schwächeren ...." (Fn. 21, Nr. 8 zu Art. 222 AEUV); ähnlich ders. (Fn. 71), S. 105. R. Streinz (Fn. 21, Nr. 3 vor Art. 27 GR-Charta) hebt vier Funktionen der unter „Solidarität“ versammelten Rechtspositionen hervor: Freiheitsrechte, Schutzansprüche, Teilhaberechte und Zielbestimmungen. Noch deutlicher bezieht M. Holoubek (in: Schwarze, Fn. 21, Nr. 8 zu Art. 27 GRC) Solidarität auf die von mir herausgearbeitete arbeitsbezogene Sozialstaatlichkeit. Im Kapitel „Solidarität“ gehe es , ,im Kern ... um ein Bündel von Rechten und Gewährleistungen, die den Menschen in einem zentralen Punkt seiner Lebensgestaltung, nämlich seiner Erwerbstätigkeit und hier - gezielt auf den wenigstens bislang für die meisten Menschen einschlägigen Fall unselbstständiger oder abhängiger Erwerbstätigkeit - schützen“. 
in der Charta und anderen internationalen Rechtsdokumenten aufgeführt und konkretisiert.

Der zweite normative Bestandteil des Solidaritätsprinzips ist in der Präambel in erforderlicher Klarheit hervorgehoben: Solidaritätsbezogene Rechte erschöpfen sich nicht in Ansprüchen, vulgo „im Nehmen“, sie sind mit Verantwortung verbunden, die den Bürger/innen kraft Gesellschaftszugehörigkeit auferlegt ist. Ihre Ausübung ist ,mit Verantwortung und mit Pflichten sowohl gegenüber den Mitmenschen als auch gegenüber der menschlichen Gemeinschaft und zukünftigen Generationen verbunden. "73 Deshalb geht Solidarität mit Umverteilungspflichten - zwischen, aber auch innerhalb von Mitgliedstaaten und Regionen, zwischen, aber auch innerhalb von Klassen und Schichten - einher. Und sie erlegt Individuen, Gruppen und Organisationen Verantwortung für gesellschaftliche Auswirkungen (besonders für „,negative Externalitäten“) ihres Verhaltens auf.

Unter diesem Aspekt macht es Sinn, dass der Lissaboner Vertrag den Artikel zum Umweltschutz (Art. 37) in den Titel ,Solidarität“ einordnet. Er nimmt damit Bezug auf Art. 191 Abs. 2 UAbs. 1 AEUV, der das ,hohe Schutzniveau“ wesentlich durch die Vermeidung negativer externer Effekte an der Quelle erreichen will: „,Die Umweltpolitik der Union ... beruht auf den Grundsätzen der Vorsorge und Vorbeugung, auf dem Grundsatz, Umweltbeeinträchtigungen mit Vorrang an ihrem Ursprung zu bekämpfen, sowie auf dem Verursacherprinzip. " Solidarische Folgenverantwortung ist nicht auf das Umweltgebiet beschränkt, sie findet auch andere Anwendungsfelder - u.a. auf dem Gebiet abhängiger Arbeit. Dort erhält sie noch durch die Querschnittsklausel des Art. 9 AEUV Relevanz: Danach müssen nämlich „Maßnahmen“ der EU in anders gelagerten Politikfeldern auch am sozialen Folgenbündel - hohes Beschäftigungsniveau, angemessener sozialer Schutz, Bekämpfung der sozialen Ausgrenzung und hohes Niveau der allgemeinen und beruflichen Bildung und des Gesundheitsschutzes - gemessen werden.

Solidarität ist somit ein dritter - historisch jüngster, aber thematisch unmittelbar einschlägiger - Bestandteil der arbeitsbezogenen europäischen Sozialstaatlichkeit.

\section{Bedeutung des Grundprinzips der Sozialstaatlichkeit neben den drei konstituierenden Werten}

Diese drei Werte - interpretiert im Lichte des Kohärenzgebotes (Art. 7 AEUV) bringen in ihrem Zusammenwirken und ihrer Ausstrahlung für das Feld abhängiger Arbeit ein normatives Grundprinzip zum Vorschein. Dieses ist sicherlich sehr differenziert, repräsentiert unterschiedliche Blickwinkel und ist in Teilen auf einem Allgemeinheitsniveau, das der Konkretisierung bedarf. Im Wesentlichen aber ist

73 So die Präambel der Grundrechte-Charta. Den Pflichtencharakter der Grundrechte betonen J. Meyer (wie Fn. 71), E. Riedel in: Meyer (Fn. 21), Nr. 32 vor Titel IV GR-Charta und H. Jarass, Charta der Grundrechte der Europäischen Union, 2. Aufl., München: Beck, Einleitung S. 9; s. auch U. Mückenberger, Artikel „Solidarität“, in: Kreft/Mielenz (Hrsg.), Wörterbuch Soziale Arbeit, 2013, 7. Aufl., S. 751-754, hier S. 752. 
dieses Grundprinzip klar und normativ bestimmt. Auf Arbeit bezogen kann man es so fassen: Arbeit darf nicht dem Geschehen des Marktes überlassen bleiben. Das Grundprinzip verlangt Maßstäbe für bewusste politische Intervention in das Marktgeschehen und stellt diese in Umrissen bereits selbst her. Die gebotene Kohärenz lässt aus den drei diskutierten Werten Menschenwürde, Demokratie und Solidarität das gemeinsame normative Ziel extrapolieren, einen gesellschaftsverträglichen und nachhaltigen Umgang mit Arbeit zu gewährleisten und dabei Schwächere materiell wie verfahrensmäßig zu stärken. Dieses Grundprinzip ähnelt durchaus dem, was im deutschen und anderen nationalen Verfassungsrechten als ,, Sozialstaatlichkeit “ bezeichnet wird. Es ist zwar im Mehrebenen-System EU nicht mit „Sozialstaatsprinzip“ gleichzusetzen - denn dieses setzt als Staatsprinzip Staatlichkeit voraus. Das Grundprinzip gilt aber auch für die EU. Es ist somit als soziale Verfasstheit im Ziel-, Aufgaben- und Befugniskatalog des Staatenverbundes EU zu verstehen, die auf mehreren Ebenen einzulösen ist und die damit der politischen Verfasstheit des Mehrebenen-Systems Rechnung trägt.

Dieses Grundprinzip europäischer sozialstaatlicher Verfasstheit hat eigenständige Berechtigung und Bedeutung. Es erschöpft sich nicht in der Summe der Geltung der drei konstituierenden Werte der Menschenwürde, Demokratie und Solidarität. Von Bogdandy hat mit dem Grundprinzip jene Normen des Primärrechts gedeutet, „die eine normativ begründende Funktion für das Ganze der Unionsrechtsordnung haben." 74 Damit stimmt auch das Grundprinzip der Sozialstaatlichkeit überein. Es macht deutlich, dass nicht die Markt- und Wettbewerbskomponente „das Ganze“, „die Identität“ der europäischen Rechtsordnung ausmacht: Eine einfache oder auch nur vorrangige Marktidentität der EU zu postulieren, wäre nach dem Durchgang durch die drei Grundwerte Menschenwürde, Demokratie und Solidarität schlicht absurd. Vielmehr ist Sozialität selbst identitätsbegründend für das Unionsrecht. Oben (unter VI. 2.) musste noch offengelassen werden, wie der in Art. 3 Abs. 3 EUV neu eingeführte Begriff der ,,sozialen Marktwirtschaft“ $\mathrm{zu}$ verstehen sei: $\mathrm{Ob}$ - normativ gesehen - das „Soziale“ konstituierend für die „Marktwirtschaft“ oder ob es ein abmilderndes Epitheton für deren Hegemonie sei. Das aufgewiesene Grundprinzip des Sozialen im Mehrebenen-System EU entscheidet nun diese Alternative im erstgenannten Sinne. Die aufgewiesene soziale Verfasstheit Europas gibt jedem Handeln jedes der Akteure der Union auf, dieses Grundprinzip zu realisieren - auch wenn dabei empfindliche Abstriche am Wettbewerb oder Marktlichen anstehen. Soziale Berechtigungen sind nicht einfach an der Elle der Grundfreiheiten zu messen. Es ist umgekehrt: Die Ausübung der Grundfreiheiten ist daran zu messen, ob und inwieweit sie den Anforderungen des Grundprinzips der Sozialstaatlichkeit als Prüfungsmaßstab entspricht oder nicht.

Sozialstaatlichkeit gibt so auch einen wesentlichen Orientierungspunkt und Auslegungsmaßstab für die Bestimmungen des europäischen Primär- und Sekundärrechts 
ab. Auf ihn stützt sich auch das bereits erwähnte, in Art. 7 AEUV verankerte Kohärenzgebot, das verlangt, dass EU-Politiken und -Maßnahmen mit ihren Zielen insgesamt konsistent sein müssen. ${ }^{75}$ Eichenhofer resümiert im Sinne eines Sozialprinzips die ,zahlreiche(n) Einzelbestimmungen im Primärrecht", die hier einschlägig sind, „namentlich diejenigen über die Ziele und Aufgaben der EU, die Nichtdiskriminierung, zwischenstaatliche Sozialrechtskoordination, Beschäftigungspolitik, Mindeststandards und die Harmonisierung sozialer Sicherheit, sozialer Dialog, Grundfreiheiten, öffentliche Monopole sowie staatliche Beihilfen."76 Schachtschneider hebt aus der Perspektive des europäischen Verfassungsrechts die hochgradige Einwirkung dessen hervor, was er das „Sozialprinzip“ nennt. Er moniert zugleich die fehlende Konsequenz der EU auf sozialpolitischem Gebiet: „Die Befugnisse der Gemeinschaft in Art. 136 ff. EGV [heute Art. 151 AEUV] hatten sich durchaus von der Theorie bestmöglicher sozialer Entwicklung durch größtmögliche Marktlichkeit entfernt, ohne freilich einer gemeinschaftlichen Sozialpolitik durchgreifende Möglichkeiten zu verschaffen."77 Kohärenz gewinnen die aufgeführten Sozial-Bestimmungen des EU-Rechts in der Tat erst, wenn sie im Kontext und als gebotener Ausdruck der durch die drei Grundwerte Menschenwürde, Demokratie und Solidarität gebildeten europäischen sozialen Verfasstheit verstanden werden.

\section{Gemeinsame internationale Abkommen und gemeinsame Verfassungstraditionen der Mitgliedstaaten}

Für dieses Ergebnis sollen zum Abschluss noch einige Indizien aus den beiden anderen u.a. in Art. 6 EUV genannten Rechtsquellen - gemeinsame internationale Abkommen und gemeinsame Verfassungstraditionen der Mitgliedstaaten - zusammengetragen werden. ${ }^{78}$ Art. 6 Abs. 3 EUV nennt die Europäischen Menschenrechtskonvention, der er durch den Beitrittszwang in Abs. 2 besonderes Gewicht verleiht. ${ }^{79}$ Zugleich erhebt Art. 6 Abs. 1 EUV die GRCh in den Rang des Primärrechts. Deren Präambel nimmt im fünften Erwägungsgrund auf die sozialen Grundrechte der MS Bezug - nämlich „,in Bestätigung der Bedeutung, die sie [die Mitgliedstaaten] den sozialen Grundrechten beimessen, wie sie in der am 18. Oktober 1961 in Turin unterzeichneten Europäischen Sozialcharta und in der Unionscharta

75 S. o. Abschn. IV.

76 So E. Eichenhofer (Fn. 16), S. 39. Es folgt auf S. 39-56 die Darstellung der Ziele, Aufgaben und Befugnis der EU auf sozialem Gebiet - wobei E. Eichenhofer sich bewusst nicht auf den engen Begriff von „Sozialrecht“ beschränkt, sondern den Arbeits- und Sozialrecht umfassenden Begriff des „droit social“ verwendet. In der Tat verlangt die Ausstrahlung des Grundprinzips der Sozialstaatlichkeit nicht eine formale, sondern eine funktionale Abgrenzung der Regelungen zugunsten abhängiger Arbeit. Nach Redaktionsschluss erschien der Aufsatz von Eberhard Eichenhofer, Soziales Europa, in: VSSR - Vierteljahresschrift für Sozialrecht 2014, H. 1, S. $29-86$. Er weist Übereinstimmungen mit meinem Gedankengang auf, kann aber hier im Detail nicht mehr berücksichtigt werden.

77 K. A. Schachtschneider (Fn. 32), S. 185. Auf den S. 181-257 findet sich eine breite Darstellung des sozialpolitischen europäischen Regelungsgeflechts.

$78 \mathrm{Zu}$ deren Bedeutung s. oben Abschnitt IV.

79 Dazu K. Lörcher (Fn. 36), S. 23-27. 
der sozialen Grundrechte der Arbeitnehmer von 1989 festgelegt sind“. Positivrechtlich findet eine erneute Bezugnahme im Primärrecht durch Art. 151 AEUV statt. Die Europäische Menschenrechtskonvention und die Europäische Sozialcharta beide aus dem Rechtskreis des Europarats - beziehen sich auf Rechte aus den Gebieten der politischen und der sozialen Verfassung, so wie es die drei genannten Grundwerte durch Hervorhebung der Demokratie einerseits, der Menschenwürde und Solidarität andererseits tun. Die Gemeinschaftscharta der Arbeitsnehmerrechte von 1989 (die zwar nicht rechtsverbindlich vereinbart wurde, gleichwohl in Art. 151 AEUV bekräftigend in Bezug genommen ist) und die GRCh - aus dem Rechtskreis von EWG/EG/EU - konkretisieren soziale Rechte und stellen sie zugleich in den Kontext von politischen Rechten und Solidarität. Hinzu tritt eine große Zahl internationaler Abkommen, denen die EU-Mitgliedstaaten zugehören oder beigetreten sind - etwa die Satzung der Vereinten Nationen (in Bezug genommen im siebten Erwägungsgrund des AEUV) und darüber die beiden UN-Pakte über politische und zivile Rechte sowie über wirtschaftliche, kulturelle und soziale Rechte und nicht zuletzt der Kanon von Übereinkommen der ILO. Auch diese Gemeinsamkeit der Mitgliedstaaten gewinnt erst Konsistenz, wenn sie nicht als vereinzelte „Vertragsparallelität“", sondern als für die EU identitätsbildende Ausprägung des Grundprinzips sozialer Verfasstheit verstanden und interpretiert werden.

Über diese Normen und Übereinkommen i.E. Rechenschaft abzulegen, liegt nicht in der Absicht dieses Beitrags. Auch für die Rechtsquelle der gemeinsamen Verfassungstraditionen der Mitgliedstaaten muss ich mich mit Hinweisen begnügen. Eine detailorientierte Darstellung erübrigt sich schon deshalb, weil dieses Kriterium in Art. 6 Abs. 3 EUV ersichtlich der Judikatur des EuGH nachvollzogen ist. Diese verlangt nicht einen ,durchschnittlichen Mittelwert“" von vielen einzelnen Verfassungsbestimmungen in den MS. Sie stellt nur das Gebot einer ,"negativen“ Kontrollfunktion" 80 auf: Dass nicht vereinzelte mitgliedstaatliche Verfassungsbestimmungen, die in Widerspruch zu denen anderer Mitgliedstaaten stehen, als gemeinsame Verfassungstraditionen missverstanden werden.

Vor diesem Hintergrund kann für die EU Einigkeit in der Anerkennung arbeitsbezogener sozialer grund- und staatsrechtlicher Ziele, Aufgaben und Befugnisse konstatiert werden. Diese nehmen in unterschiedlicher Weise Bezug auf die drei konstituierenden Werte von Sozialstaatlichkeit und damit auf diese selbst. Ich folge hier der hilfreichen Aufstellung bei Hufeld/Epiney und reichere sie um die Beispiele von Peter Häberle an, die sogar für universelle Geltung von Teilen dieser Rechte sprechen. ${ }^{81}$

Hufeld/Epiney führen ausschließlich MS bzw. Mitglieder des Europäischen Wirtschaftsraums (EWR) auf. Belgiens „koordinierte Verfassung Belgiens“ z.B. führt

80 R. Streinz in: Streinz (Fn. 21); dazu bereits oben IV.

81 U. Hufeld/A. Epiney, Europäisches Verfassungsrecht. Vertragliches Europaverfassungsrecht. Staatliches Verfassungsrecht, 2010, 2. Aufl., S. 229-634; Weitere Beispiele P. Häberle (Fn. 7), S. 556 u.ö. und bei A. Hatje (Fn. 36), Art. 6 EUV, Fn. 15 ff. 
in Art. 7 auf: „,... verfolgen ...die Ziele einer nachhaltigen Entwicklung in deren sozialen, wirtschaftlichen und umweltbezogenen Aspekten unter Berücksichtigung der Solidarität zwischen den Generationen“. Art. 23 erwähnt neben dem „Recht, ein menschenwürdiges Leben zu führen", das Recht auf Arbeit und gerechte Arbeitsbedingungen, soziale Sicherheit und Gesundheitsschutz, das Recht auf Information, Konsultation und Kollektivverhandlungen. Frankreichs „,Verfassung der französischen Republik“ erwähnt in ihrer Präambel die „Verbundenheit mit den Menschenrechten“ gemäß Erklärung von 1789, ,welche durch die Präambel der Verfassung von 1946 bestätigt und ergänzt wurde“" Diese sieht soziale Rechte vor: Arbeit (Abs. 3), Kollektivvertretung und Streikrecht (Abs. 4-6), Schutz der Entwicklung des „Einzelnen“ und der „Familie“, Gesundheitsschutz, bei Arbeitsunfähigkeit „Unterhalt“ (Abs. 8/9). Italiens „Verfassung der Italienischen Republik“ erklärt in Art. 1 Abs. 1: „Italien ist eine demokratische, auf die Arbeit gegründete Republik.“ Art. 3 erwähnt die Gleichheit und die Aufgabe der Republik, tatsächliche Hindernisse für Freiheit und Gleichheit zu beseitigen (,,volle Entfaltung der menschlichen Persönlichkeit und der wirksamen Teilhabe aller Arbeiter an der politischen, wirtschaftlichen und sozialen Gestaltung des Landes"'). Art. 18/19 erheben Versammlungs-/Vereinigungsfreiheit, Art. 35-40 den Schutz der Arbeit, sozialstaatliche Einrichtungen, Gewerkschaftsfreiheit und Streikrecht zum Gebot. Die ,soziale Funktion" des Privateigentums wird in Art. 42 Abs. 2 zum Gebot erhoben.

In den Niederlanden und ihrer „Verfassung des Königreiches der Niederlande“ gebieten Art. 8/9 die Vereinigungs-/Versammlungsfreiheit, Art. 15 Arbeitsbeschaffung und Arbeitsschutz sowie Art. 16 die Existenzsicherung und Sozialhilfe, Art. 20 gebieten die „Verteilung des Wohlstandes“ und „soziale Sicherheit“. In Österreich schreibt das „Bundes-Verfassungsgesetz der Republik Österreich“"82 in Art. 12 die Versammlungs-/Vereinigungsfreiheit und in Art. 18 die Berufsfreiheit fest. Polen normiert in der „Verfassung der Republik Polen“ in Art. 12 auch die Gewerkschaftsfreiheit („Gründung und Tätigkeit“) und legt in Art. 20 fest: „Die soziale Marktwirtschaft, gestützt auf die Freiheit der wirtschaftlichen Tätigkeit, das Privateigentum und die Solidarität, den Dialog und die Zusammenarbeit der Sozialpartner, bildet die Grundlage der wirtschaftlichen Ordnung der Republik Polen.“ Art. 24 schreibt darüber hinaus den Arbeitsschutz und die staatliche Arbeitsaufsicht vor. Art. 30 konstatiert: Die Menschenwürde ,,bildet die Quelle der Freiheiten und Rechte des Menschen und des Bürgers“. Art. 57/58 erklären die Vereinigungs-/ Versammlungsfreiheit, Art. 59 die Koalitionsfreiheit und Streikrecht der Gewerkschaften. Art. 65-68 die Berufsfreiheit, Mindestlohn und Vollbeschäftigungspolitik, den Arbeits- und Gesundheitsschutz sowie Soziale Vorsorge und Hilfe zum Gebot. Ähnlich konkret - um ein Beispiel aus einem europäischen Staat zu nennen, der nicht Mitglied der EU ist - ist die „Bundesverfassung der Schweizerischen Eidge-

82 Laut Art. 149 Abs. 1 des „Bundes-Verfassungsgesetzes der Republik Österreich“, zuletzt geändert am 30.12.09 gilt als „Verfassungsgesetz“ i. S. d. Art. 44 Abs. 1 auch das „Staatsgrundgesetz v. 21. Dezember 1867“ mit Rechten der Staatsbürger. 
nossenschaft““. Bereits die Präambel verkündet: „... gewiss, dass nur frei ist, wer seine Freiheit gebraucht, und dass die Stärke des Volkes sich misst am Wohl der Schwachen.“ Art. 2 legt auf, ,die gemeinsame Wohlfahrt, die nachhaltige Entwicklung, den inneren Zusammenhalt und die kulturelle Vielfalt des Landes“ zu fördern und sorgt „für eine möglichst große Chancengleichheit unter den Bürgerinnen und Bürgern." Art. 7 nimmt die Menschenwürde als Ausgangspunkt. Art. 8 Abs. 3 sorgt für rechtliche und tatsächliche Gleichstellung von Frauen ,vor allem in Familie, Ausbildung und Arbeit.“. Art. 12 begründet Recht auf Hilfe in Notlagen Art. 22/23 und 28 die Versammlungs-, Vereinigungs- und Koalitionsfreiheiten (mit Streik- und Aussperrungsrecht) und Art. 35 die staatliche Bindung an die Grundrechte. Das 3. Kapitel heißt „Sozialziele“- Art. 41 verpflichtet zu sozialer Sicherheit, Pflege, Lebensunterhalt durch Arbeit, Art. 108 zu Arbeitsschutz und Kollektivvereinbarungen, Art. 111-118 zu Risikovorsorge als Grundrechten und Staatszielen.

Ähnlich proklamiert die ,Verfassung des Königreichs Spanien“ schon in ihrer Präambel: „das Wohl aller ihrer Bürger zu fördern“, eine ,gerechte Wirtschafts- und Sozialordnung“ sowie „würdige Lebensverhältnisse für alle zu sichern“. Art. 1 Abs. 1 bestimmt: „Spanien konstituiert sich als demokratischer und sozialer Rechtsstaat und bekennt sich zu Freiheit, Gerechtigkeit, Gleichheit und politischem Pluralismus als obersten Werten seiner Rechtsordnung". Das Sozialstaatsprinzip wird sodann konkretisiert. Art. 7 legt die Gewerkschaftsfreiheit fest, weiter dann Art. 28 mit Gewerkschaftsfreiheit und ,Streikrecht der Arbeitnehmer zur Verteidigung ihrer Interessen“. Art. 10 nimmt Menschenwürde als Ausgangspunkt und fordert eine Auslegung der Grundrechte in Übereinstimmung mit der Allgemeinen Erklärung der Menschenrechte und mit von Spanien ratifizierten internationalen Verträgen und Abkommen. Art. 21/22 erklären die Versammlungs-/Vereinigungsfreiheit, Art. 35 die Berufsfreiheit und Recht auf Arbeit, Art. 37 das Recht auf Kollektivverhandlungen, Art. 40 die Vollbeschäftigungs- und Arbeitsschutzpolitik, Art. 41 die Risikovorsorge durch soziale Sicherung, Art. 43 den Gesundheitsschutz und schließlich Art. 53 die Grundrechtsbindung hinsichtlich der in Art. 14 bis 38 enthaltenen Rechte. Den „Verbraucherschutz“ gebietet Art. 51 der spanischen Verfassung.

Tschechien - um zwei weitere Beispiele der mittel-/osteuropäischen MS zu geben - erklärt in der „Verfassung der Tschechischen Republik“ von 2009 (Art. 3) die Charta der Grundrechte und Grundfreiheiten in der Fassung von 1998 zum Bestandteil der Verfassungsordnung. Diese proklamiert in Art. 1 und Art. 10 die Menschenwürde und erklärt in Art. 19/20 die Versammlungs-/Vereinigungsfreiheit, in Art. 26 das Recht auf Lebensunterhalt durch Arbeit, sonst staatliche Unterstützung, in Art. 27 die Gewerkschaftsfreiheit und das Streikrecht, in Art. 28-31 den Arbeitsund Gesundheitsschutz, den gerechten Lohn und eine materielle Risikovorsorge für verpflichtend. „Die Verfassung der Republik Ungarn“ von 2009 schließlich proklamiert in ihrer Präambel die „soziale Marktwirtschaft". $§ 4$ bekennt sich zur gewerkschaftlichen Schutzfunktion. § 9 erklärt zwar zur „Wirtschaftsform“ die Markt- 
wirtschaft, stellt ihr aber sogleich in $\S 17$ die Fürsorge für Bedürftige, in $\S 54$ das Recht auf Leben und Menschenwürde, in $\S \S 62 / 63$ die Versammlungs-/Vereinigungsfreiheit, in $\S \S 66 \mathrm{u}$. 70/A die Gleichheit einschließlich positiver Diskriminierung, in $\S 70 / \mathrm{B}$ das Recht auf Arbeit und Arbeitsschutz, in $\S 70 / \mathrm{D}$ das Recht auf Gesundheitsschutz, in $\S 70 / \mathrm{E}$ das Recht auf soziale Sicherung sowie in $\S 70 / \mathrm{C}$ die Koalitionsfreiheit und das Streikrecht zur Seite.

Die folgenden Beispiele bei Häberle unterstreichen diesen Eindruck. ${ }^{83}$ Art. 80 lit. a der Verfassung Portugals fordert die „Unterordnung der wirtschaftlichen Macht unter demokratische Staatsgewalt“, Art. 81 lit. e das Verbot des „Missbrauchs wirtschaftlicher Macht". Irland erhebt in Art. 45 Abs. 4 Nr. 1 seiner Verfassung von 1937/72 zu Gebot: „Der Staat gelobt, die wirtschaftlichen Interessen der schwächeren Gruppen der Gemeinschaft mit besonderer Sorgfalt zu schützen, sowie, wo es notwendig ist, zum Unterhalt der Kranken, Witwen, Waisen und Alten beizutragen". Griechenland bekennt sich in Art. 23 Abs. 2 seiner Verfassung zu Gewerkschaftsfreiheit und Streikrecht. In Litauen ist Abschnitt IV der Verfassung überschrieben mit „Volkswirtschaft und Arbeit" und proklamiert darin, dass ,wirtschaftliche Freiheiten und soziale Schutzrechte in einen geglückten Zusammenhang geraten". 84

Man könnte zahlreiche weitere Beispiele aus den MS hinzufügen. Die Beispiele sollen hier lediglich hinlängliche Indizien dafür abgeben, dass sozialstaatliche Traditionen einen Grundzug europäischer Staatlichkeit und seiner Verfasstheit bilden. Gegenbeispiele gegen Sozialstaatlichkeit fehlen gänzlich. Erst sie würden - im Sinne des EuGH und seines Prüfungsmaßstabes der ,negativen Kontrollfunktion“ - zu Vorsicht gegenüber der Annahme gemeinsamer europäischer Verfassungstraditionen Anlass geben. ${ }^{85}$

\section{Normative Kontur des sozialstaatlichen Grundprinzips im Verfassungsgefüge des europäischen Mehrebenen-Systems}

Nach Lage der gemusterten rechtlichen Quellen kann ein Grundprinzip europäischer Sozialstaatlichkeit als Sozialverfassung nicht ernsthaft negiert werden. Gewiss muss man die Sensibilität für eine große Diversität unter den MS bewahren. Man muss sich außerdem der Tatsache bewusst bleiben, dass ein Grundprinzip - wie das hier dargestellte der europäischen Sozialstaatlichkeit - in praktischer Konkordanz mit anderen gleichrangigen Zielen und Aufgaben der EU gedacht und interpretiert werden muss. ${ }^{86}$ Dies ändert aber nichts an der Existenz des Grundprinzips europäischer Sozialstaatlichkeit.

86 Vgl. R. Bieber in: Bieber/Epiney/Haag, Die Europäische Union. Europarecht und Politik, 2011, 9. Aufl., zu Art. 3: Der Vertrag von Lissabon habe durch Art. 3 I u. III EUV eine Abkehr von der Wirtschaftstheorie der liberalen Marktwirtschaft (,freier unverfälschter Wettbewerb“) vollzogen (allerdings noch Art. 120 AEUV) und 
Dieses kann abschließend wie folgt umrissen werden:

Im Zentrum der auf Arbeit bezogenen europäischen Sozialverfassung steht die der Menschenwürde entsprechende individuelle und kollektive Selbstbestimmung arbeitender Menschen über die eigene Lebensführung, die berufliche Einmündung und die Vereinbarung beruflicher und außerberuflicher Aktivitäten. Flankiert wird Selbstbestimmung durch gebotenen Schutz vor Notlagen und Zwängen, innerhalb wie außerhalb des Erwerbslebens, durch kollektive Stimme und Beteiligung, durch Menschenwürde sichernde Standards sowie durch Chancen- und Ressourcenumverteilung auf solidarischer Grundlage. Sozialität wird den am Arbeitsleben Beteiligten durch Solidarität auch insoweit abverlangt, als sie für soziale Folgen ihrer Tätigkeit Verantwortung und auch für die Bedingungen der Selbstverwirklichung anderer Sorge zu tragen haben.

Allererste Voraussetzung der auf Selbstbestimmung, Schutz und Verantwortung bauenden europarechtlichen Sozialstaatlichkeit ist ein, heute nicht mehr als selbstverständlich zu unterstellender, Primat demokratischer Politik. Auch bezogen auf das Arbeitsleben muss eine demokratisch legitimierte und effektive europäische Politik die Hegemonie gegenüber der sich europäisierenden und globalisierenden Ökonomie einnehmen bzw. wiedergewinnen. ${ }^{87}$ Der Staatsbezug dieser hegemonialen Politik folgt aber selber noch dem Gebot der Selbstbestimmung der unmittelbar Beteiligten und ist daher in mehrfacher Weise relativiert - er erkennt die Rollen von Staaten und Staatsverbund im Mehrebenen-System an, er verankert politische Willensbildung bei nicht-staatlichen Akteuren und ermutigt und befähigt proaktiv-subsidiär nicht-staatliche problem- und lösungsnahe Akteure zu gesellschaftlich verantwortlicher Selbstregulierung.

Natürlich stellt sich nach den hier vorgestellten Überlegungen die Aufgabe, das Mehrebenen-System EU im Hinblick auf die gebotene sozialstaatliche Aufgabenallokation zu mustern und zu systematisieren. Mit meinem Nachweis einer europäischen Sozialstaatlichkeit ist noch keine Antwort auf die Fragen gegeben, wer welche Aufgaben auf welcher Ebene zu erfüllen hat, welche Rolle dem Subsidiaritätsprinzip zu kommt usw. Ich konnte und wollte zu dieser Frage lediglich eine Vorfrage beantworten: Nämlich ob im europäischen Mehrebenen-System lediglich die MS ihrer jeweiligen sozialstaatlichen Verpflichtung unterliegen oder ob auch die EU selbst einer primärrechtlichen sozialstaatlichen Verpflichtung unterliegt. Der Beitrag wollte der letztgenannten Annahme Plausibilität geben.

durch das Prinzip der wettbewerbsfähigen sozialen Marktwirtschaft ersetzt sowie dieses durch die „Querschnittsklausel des Art. 9 AEUV“" ergänzt (S. 99/100). Ähnlich betont T. Oppermann, in: Oppermann/Classen/ Nettesheim (Fn. 22), in: Art. 3 EUV - ,flankiert“ durch die sozialpolitische Querschnittsklausel Art. 9 AEUV - gegenüber der „offenen Marktwirtschaft mit freiem Wettbewerb“ (Art. 4 EGV-Nizza) (S. 338). Später erfolgt der Hinweis auf das dadurch konstituierte „europäische Sozialmodell“ (S. 495).

87 Zur derzeitigen Bedrohung des Primats demokratischer Politik im Angesicht der zunehmenden Macht globalisierter ökonomischer Akteure aus juristischer Sicht, s. A. Supiot, L'esprit de Philadelphie. La justice sociale face au marché total, 2010; aus sozialwissenschaftlicher Sicht $W$. Streeck (Fn. 60). 


\section{Formulierungsversuch für ein auf Arbeit bezogenes europäisches sozialstaatliches Grundprinzip}

Selbstbestimmung. Menschen haben - in welcher sozialen, geographischen, familiären, Bildungs-, geschlechtlichen u.a. Lebenslage sie sich auch befinden - in Bezug auf Arbeit ein individuelles und kollektives Recht auf Selbstbestimmung. Dies betrifft das Recht auf einen würdigen Lebensunterhalt innerhalb wie außerhalb einer Erwerbsarbeit, die Freiheit einer Erwerbsaufnahme, das Recht, die Befähigung zum Erwerb zu erlangen, die Freiheit und Gleichheit in der Arbeit, die Vereinbarkeit der Arbeit mit sonstigen Lebensbelangen, die Freiheit zur Beendigung eines Arbeitsverhältnisses. Es umfasst die Möglichkeit, kollektive Interessenvertretung zu bilden und sich in ihr zu betätigen, wie auch das Recht der Kollektivvereinigung, sich zu bilden, zu existieren und sich zu betätigen.

Sozialer Schutz. Menschen haben - in welcher sozialen, geographischen, familiären, Bildungs-, geschlechtlichen u. a. Lebenslage sie sich auch befinden - in Bezug auf Arbeit ein Recht auf individuellen und kollektiven Schutz. Sie werden innerhalb von Arbeitsbeziehungen vor von ihnen unbeeinflussbaren Risiken bewahrt und bei ihrer Bewältigung unterstützt. Sie erlangen Schutz vor schädigenden und entwürdigenden Arbeitsbedingungen, vor Diskriminierung und Ausgrenzung durch einen verlässlichen Rahmen von Sicherheit in der Arbeitsbeziehung. Innerhalb wie außerhalb von Arbeitsbeziehungen kommt Menschen eine Risikovorsorge zuteil, die Schädigungen möglichst zuvorkommt, die eine effektive Risikobewältigung erlaubt, ohne die Selbstbestimmungsfähigkeit einzuschränken, und die eine würdige und gesellschaftsintegrative Einkommensgrundlage bietet.

Verantwortung. Staatliche und nicht-staatliche Instanzen sorgen dafür, dass Arbeitsleben und außererwerbliche Lebensverhältnisse gut neben- und miteinander bestehen können. Sie fördern Lebensverhältnisse, Infrastruktur- und sonstige gesellschaftliche Rahmenbedingungen, die gute Arbeit ermöglichen und erleichtern. Sie treten Auswirkungen von Arbeit entgegen, die die gesellschaftlichen und natürlichen Lebensgrundlagen sowie den sozialen Zusammenhalt gefährden.

Demokratische Gestaltung. Die europäische Sozialstaatlichkeit sicherzustellen, ist eine demokratisch zu legitimierende politische Gestaltungsaufgabe, für die Politik den nötigen Freiraum gegenüber der Ökonomie besitzen muss. An der Erfüllung dieser politischen Aufgabe wirken die EU und die Mitgliedstaaten in geeignetem und effektivem Zusammenwirken im Mehrebenen-System mit. Sie übertragen die Bewältigung geeigneter sozialstaatlicher Aufgaben auf sachnahe nicht-staatliche Akteure und Organisationen sowie auf privat-öffentliche Kooperationen. Dabei tragen sie aktiv dazu bei, dass nicht-staatliche Akteure und Organisationen zur gebotenen sozialstaatlichen Aufgabenerfüllung bereit, befähigt und in der Lage sind bzw. werden. 